\title{
ON THE CLASSIFICATION OF IRREGULAR SURFACES OF GENERAL TYPE WITH NONBIRATIONAL BICANONICAL MAP
}

\author{
FABRIZIO CATANESE, CIRO CILIBERTO, AND MARGARIDA MENDES LOPES
}

\begin{abstract}
The present paper is devoted to the classification of irregular surfaces of general type with $p_{g} \geq 3$ and nonbirational bicanonical map. Our main result is that, if $S$ is such a surface and if $S$ is minimal with no pencil of curves of genus 2, then $S$ is the symmetric product of a curve of genus 3 , and therefore $p_{g}=q=3$ and $K^{2}=6$.

Furthermore we obtain some results towards the classification of minimal surfaces with $p_{g}=q=3$. Such surfaces have $6 \leq K^{2} \leq 9$, and we show that $K^{2}=6$ if and only if $S$ is the symmetric product of a curve of genus 3. We also classify the minimal surfaces with $p_{g}=q=3$ with a pencil of curves of genus 2, proving in particular that for those one has $K^{2}=8$.
\end{abstract}

\section{INTRODUCTION}

It is well known that the bicanonical map of a curve of genus $g \geq 2$ fails to be birational if and only if $g=2$. This exception reproduces itself in dimension 2; namely if a surface $S$ of general type has a pencil of curves of genus 2 , i.e. it has a rational map to a curve whose general fibre $F$ is irreducible of genus 2 , then the line bundle $\mathcal{O}_{S}\left(K_{S}\right) \otimes \mathcal{O}_{F}$ is special on $F$, and therefore the bicanonical map $\phi$ of $S$ cannot be birational.

We shall refer to the above exception to the birationality of the bicanonical map $\phi$ as the standard case. A non-standard case will be the case of a surface of general type $S$ for which $\phi$ is not birational, but there is no pencil of curves of genus 2 . Bombieri (see [B], thm. 5) proved that if $K^{2} \geq 10$ and $p_{g} \geq 6$, then all surfaces for which $\phi$ is not birational present the standard case. He also gave an example (see [B], pg. 194), already found by Du Val (see [D]), of a minimal surface with $K^{2}=9, p_{g}=6$ exhibiting the non-standard case. Later on I. Reider proved in $[\mathrm{R}]$ that the hypothesis $K^{2} \geq 10$ alone ensures that one has a standard case if $\phi$ is not birational.

From these results it follows that there are only a finite number of families of minimal surfaces of general type presenting the non-standard case. It is a general paradigm in the theory of surfaces that one has good results holding for surfaces with sufficiently high values of the numerical invariants $K^{2}$ and $p_{g}$. On the other hand, any theory must include results holding for many concrete interesting cases, and most frequently the surfaces one can encounter have small values of the numerical

Received by the editors February 22, 1996.

1991 Mathematics Subject Classification. Primary 14J29.

(C)1998 American Mathematical Society 
invariants. It therefore makes sense to try to completely classify the non-standard cases.

In the case, much more extensively treated by the classical geometers, of regular surfaces, i.e. those surfaces $S$ with $q:=h^{1}\left(S, \mathcal{O}_{S}\right)=0$, an attempt to classify the non-standard cases with $p_{g} \geq 2$ was made by Du Val in [D]. In the recent paper $[\mathrm{CFM}]$ the non-standard cases with $p_{g} \geq 4$ were completely classified, essentially confirming Du Val's list. In particular, if $p_{g} \geq 4$ all non-standard cases have $q=0$.

Also Xiao Gang (see [X1]) studied the general problem of classifying the nonstandard cases, by taking the point of view of the projective study of the image of the bicanonical map. He thus determined some properties and found a list of numerical possibilities for the invariants of the cases which might occur.

The present article is devoted to the classification of the non-standard cases for irregular surfaces, and this is our main result:

Theorem A. Let $S$ be a minimal irregular surface with $p_{g} \geq 3$ for which the bicanonical map is not birational. Then $S$ presents the non-standard case if and only if $S$ is isomorphic to the symmetric product of a smooth irreducible curve of genus 3 . In particular, $p_{g}=q=3$ and $K^{2}=6$.

Indeed, concerning surfaces with $p_{g}=q=3$ we obtain here some steps toward their classification, namely:

Theorem B. Let $S$ be a minimal surface with $p_{g}=q=3$. Then $6 \leq K^{2} \leq 9$, and $K^{2}=6$ if and only if $S$ is isomorphic to the symmetric product of a smooth irreducible curve of genus 3. Moreover if $S$ admits a morphism $f: S \rightarrow B$ to a smooth curve of genus $b \geq 1$ with connected fibres of genus $g$, i.e. a so-called irrational pencil, then either $b=1$ and $g \geq 3$, or $b=g=2$ and $S$ has an unramified double cover which is the product of two smooth irreducible curves of genera 2 and 3. In the last case $K^{2}=8$, and this is the only standard case for $p_{g}=q=3$.

Concerning the non-standard case for irregular surfaces, the only other example we know of is the case of the double covering of a principally polarized abelian surface $(A, \Theta)$ branched along a divisor algebraically equivalent to $2 \Theta$. We consider it very likely that this might be the only non-standard case for irregular surfaces with $p_{g}=2$.

The organization of the paper is as follows. In $\S 0$ we recall the many results and techniques which are systematically used in the proof but are not, in our opinion, necessarily well known to the algebraic geometer in the street. In particular we give complete proofs of some results about continuous systems of curves of low selfintersection on a surface, which are perhaps known to the experts but for which we could not find an appropriate reference. We mention that these results, which turn out to be essential to handle the cases $q=1,2$, seem to be susceptible of extensions and developments. In $\S 1$ we analyse the minimal non-standard cases $S$ with $K^{2}=9, p_{g} \geq 3$, proving that such an $S$ is isomorphic to the Du Val-Bombieri surface, which has $q=0$. We use here a combination of Reider's method, as presented by Beltrametti and Sommese in [BS], and a more refined analysis carried out in $[\mathrm{CFM}]$. In $\S 2$ we prove that for $q=1,2$ only the standard case can occur. The main idea here is to consider the paracanonical system of the surface $S$, i.e. the family of curves which are algebraically equivalent to a canonical divisor of $S$, and the two related continuous systems given respectively by the fixed part $F_{\eta}$ and the movable part $\left|M_{\eta}\right|$ of $\left|K_{S}+\eta\right|$, for $\eta \in P i c^{0}(S)$. Here we use the generic 
vanishing of $[\mathrm{GL}]$ as well as the aforementioned results on continuous systems of low self-intersections, to deduce that, if $\phi$ is not birational, there is a pencil of curves of genus 2 on the surface.

In $\S 3$ we concentrate on surfaces $S$ with $p_{g}=q=3$, with particular attention to the structure of the Albanese map $a: S \rightarrow A l b(S)$. Standard inequalities concerning irrational pencils lead to the classification of those existing in our case. A key ingredient is proposition (3.1) relating the base point scheme for $H^{0}\left(S, \Omega_{S}^{i}\right)$, $i=1,2$. In the case where $K^{2}=6$ it follows that the map $a$ is unramified on the canonical model of $S$ (the proof is an easy consequence of proposition (3.1) if the canonical model is smooth; it is slightly more complicated otherwise). At this point we apply a beautiful theorem of Debarre's extending to abelian varieties the well known Fulton-Hansen connectedness theorem (see [De2]). Debarre's result ensures that $a$ is an embedding on the canonical model, whence the first part of theorem B follows easily. The remaining cases $p_{g}=q=3, K^{2}=7,8$, need a more intricate analysis, which, roughly speaking, is divided into the following steps:

(i) The case when there is a fixed part $F$ of $\left|K_{S}\right|$ can be excluded by a combinatorial and geometric analysis relying on the fact that rational curves on $S$ are contained in $F$ and they are very few (see [Mi]).

(ii) If $\left|K_{S}\right|$ has no fixed component, then the bicanonical map has degree 2 and $K^{2}=8$, and the base point scheme of $\left|K_{S}\right|$ has degree 4 . We use both the base point scheme and the involution provided by the bicanonical map in order to prove the existence of linear pencils of reducible canonical curves.

(iii) Finally we prove that the existence of these canonical pencils contradicts the geometrical information which we get from the Albanese morphism.

We should make one final comment on the differences between the irregular and the regular case. As the present paper illustrates, quite a variety of techniques must be used and are necessary in the irregular case. This contrasts with the regular case, where, as we shall see in a forthcoming paper, since the bicanonical system cuts out a complete series on the canonical curves, it suffices, for classification purposes, to establish the existence of pencils of reducible canonical curves.

\section{ACKNOWLEDGments}

The present collaboration took place in the framework of HCM contract AGE, no ERBCHRXCT940557. The paper was written while the first two authors were Professore distaccato at the Centro Interdisciplinare "B. Segre" of the Accademia Nazionale dei Lincei. Financial support has been provided by GNSAGA of CNR of Italy and by CAUL of Portugal.

\section{NOTATION AND CONVENTIONS}

We will denote by $S$ a projective algebraic surface over the complex field. Usually $S$ will be smooth, minimal, and of general type.

We denote by $K_{S}$, or simply by $K$ if there is no possibility of confusion, a canonical divisor on $S$. As usual, for any sheaf $\mathcal{F}$ on $S$, we denote by $h^{i}(S, \mathcal{F})$ the dimension of the cohomology space $H^{i}(S, \mathcal{F})$, and by $p_{g}$ and $q$ the geometric genus and the irregularity of $S$.

By a curve on $S$ we mean an effective, nonzero divisor on $S$. We denote by $p_{a}(C)$ the arithmetic genus of $C$. We denote by $C \cdot D$ the intersection number of the divisors $C, D$ on $S$, and by $C^{2}$ the self-intersection of the divisor $C$. We denote 
by $\equiv$ the linear equivalence for divisors on $S$ and by $\sim$ the numerical equivalence. $|D|$ will be the complete linear system of the effective divisors $D^{\prime} \equiv D$, and $\phi_{D}$ : $S \rightarrow \mathbf{P}\left(H^{0}\left(S, \mathcal{O}_{S}(D)\right)^{\vee}\right)=|D|^{\vee}$ the natural rational map defined by $|D|$. More generally, if $\mathcal{L}$ is a line bundle on a variety $X$ and $V \subset H^{0}(X, \mathcal{L})$ is a nonzero subspace, we denote by $|V|$ the linear system determined by $V$, and by $|\mathcal{L}|$ the complete linear system determined by $\mathcal{L}$.

A base point $p$ of a linear system $\mathcal{C}$ on a smooth surface $S$ is said to be a base point of multiplicity $h$, or an $h$-tuple base point, if the general curve of $\mathcal{C}$ has multiplicity $h$ at $p$. If $h=1$, we say that $p$ is a simple base point.

If $p$ is a simple base point for $\mathcal{C}$, it is said to be of species $k$, if two general curves of $\mathcal{C}$ have at $p$ multiplicity of intersection equal to $k$. In other words, $p$ is of species $k$ if and only if $\mathcal{C}$ has $k-1$ successive base points infinitely near to $p$. If $k=1$, we say that $p$ is simple and ordinary. Note that by blowing up a simple base point $p$ of species $k \geq 2$ of a linear system $\mathcal{C}$ on a surface $S$ and by taking the strict transform $\mathcal{C}^{\prime}$ of $\mathcal{C}$ on the blown up surface $S^{\prime}$, the linear system $\mathcal{C}^{\prime}$ has a simple base point $p^{\prime}$ of species $k-1$ lying on the exceptional divisor $E$ of $S^{\prime}$ corresponding to $p$.

We will denote by $\phi$ the bicanonical map $\phi_{2 K}$. We will set $\Sigma:=\phi(S)$ and, when $\Sigma$ is a surface, we will denote by $\sigma$ the degree of the map $\phi: S \rightarrow \Sigma$. If $\eta$ is a point in $\operatorname{Pic}(S)$ and $D$ is a divisor on $S$, we will briefly denote by $D+\eta$ any divisor corresponding to the line bundle $\mathcal{O}_{S}(D) \otimes \eta$. A divisor $D$ is numerically effective, briefly nef, if $D \cdot C \geq 0$ for every curve $C$ on $S$. We denote by $\omega_{X}$ the dualizing sheaf for any variety $X$. We will denote by $A l b(S)$, or simply by $A$ if no confusion arises, the Albanese variety of $S$ and by $a: S \rightarrow A$ the Albanese morphism.

The symbol $\simeq$ denotes in general an isomorphism between objects under consideration.

\section{Auxiliary Results}

In the present section we are going to recall a few facts which will be used in the rest of the paper.

Let $S$ be a smooth projective surface, $B$ a smooth projective curve of genus $b$. A surjective rational map $f: S \rightarrow B$ with connected fibres is called a pencil of genus $b$, or a genus $b$ pencil, of curves on $S$. We refer to $B$ as the base of the pencil and to the fibres of $f$ as the curves of the pencil. We recall that all the curves of the pencil are linearly equivalent if and only if $b=0$, in which case the pencil is called a rational pencil. If $b>0$ one says that the pencil is an irrational pencil, and in this case $f$ is a morphism. In particular, if $b=1$ one says that the pencil is an elliptic pencil. We will usually denote by $g$ the arithmetic genus of the fibres of $f$, and we will say that $f: S \rightarrow B$ is a pencil of curves of genus $g$.

(0.1) (Xiao, [X2]). Let $S$ be a minimal surface of general type and let $|K|$ be composed with a pencil of genus $b$. Then either $b=q=1$ or $b=0$ and $q \leq 2$.

(0.2) (Xiao, [X3]). Let $S$ be an irregular surface with Kodaira dimension $\kappa \geq 0$. If $S$ has a positive-dimensional linear system of curves whose general element is irreducible of geometric genus $g$, then $2 q \leq g+1$.

(0.3) (Pirola, $[\mathrm{P}]$ ). Let $S$ be an irregular surface whose Albanese image is a surface. Then there is no rational system of hyperelliptic curves on $S$.

(0.4) (Debarre, [De1]; Horikawa, [Ho]). Let $S$ be a minimal irregular surface of general type. One has: 
(i) $K^{2} \geq 2 p_{g}$;

(ii) if $K^{2} \leq 3 \chi\left(\mathcal{O}_{S}\right)-1$ [resp. if $K^{2}<\frac{8}{3} \chi\left(\mathcal{O}_{S}\right)$ ] then the Albanese map a:S $\rightarrow$ Alb $(S)$ factors through an irrational pencil $f: S \rightarrow B$ of hyperelliptic curves of genus $g \leq 3$ [resp. g=2].

(0.5) (Green-Lazarsfeld, [GL]; Beauville, [Be2]). Let $S$ be a minimal irregular surface of general type. One has:

(i) if the image of the Albanese map $a: S \rightarrow \operatorname{Alb}(S)$ is a surface and $\eta \in P i c^{0}(S)$ is a general point, then $h^{1}(S, \eta)=0$;

(ii) if $q$ is odd and on $S$ there is no irrational pencil of curves of genus $g>\frac{q}{2}$, then for a general curve $C \in|K|$ one has $h^{0}\left(C, \mathcal{O}_{C}\left(K_{S}\right)\right)=p_{g}$

(0.6) (Beauville, [Be1], pg. 343; Barth-Peters-van de Ven, [BPV], pg. 97). Let $S$ be a minimal surface and let $f: S \rightarrow B$ be a genus b pencil of curves of genus $g$. Then

$$
K^{2} \geq 8(g-1)(b-1)
$$

equality holding only if the curves of the pencil have constant modulus. Moreover,

$$
c_{2}(S)=4(g-1)(b-1)+\sum_{F}(e(F)+2 g-2),
$$

where $F$ varies among the curves of the pencil, $e(F)$ is the topological EulerPoincaré characteristic of $F$, one has $e(F)+2 g-2 \geq 0$, and equality holds, in case $g \geq 2$, if and only if $f$ is smooth along F. Finally,

$$
q \leq g+b,
$$

and equality holds if and only if $S$ is birationally equivalent to the product of $B$ with the general fibre of $f$.

(0.7) Lemma. Let $S$ be a minimal surface of general type with nonbirational bicanonical map, presenting the non-standard case. If $S$ has an irrational pencil $f: S \rightarrow B$ of curves of genus $g$, then $B$ has genus 1 and $g \geq 3$.

Proof. By Reider's results in $[\mathrm{R}]$, we may assume that $K^{2} \leq 9$. The assertion follows from (0.6) and the fact that $g \geq 3$, since we are assuming we are in the non-standard case. $\diamond$

(0.8) (Miyaoka, [Mi]). If $S$ has nef canonical divisor and $Z_{1}, \ldots, Z_{h}$ are disjoint fundamental cycles on $S$, then

$$
\sum_{i=1}^{h} m\left(Z_{i}\right) \leq 3 c_{2}-K^{2},
$$

where, with the usual notation for the type of a fundamental cycle (see [BPV], pg. 77), one has

$$
\begin{gathered}
m\left(A_{r}\right)=3(r+1)-\frac{3}{r+1}, \quad m\left(D_{r}\right)=3(r+1)-\frac{3}{4(r-2)}, \quad r \geq 4, \\
m\left(E_{6}\right)=21-\frac{1}{8}, \quad m\left(E_{7}\right)=24-\frac{1}{16}, \quad m\left(E_{8}\right)=27-\frac{1}{40} .
\end{gathered}
$$


(0.9) (Ramanujam, [Ra]; Catanese, [Ca], remark 6.8). Let D be an effective, 1-connected curve on a smooth surface. If $D$ has arithmetic genus $g<q$, by the exact sequence

$$
0 \rightarrow H^{1}\left(S, \mathcal{O}_{S}(-D)\right) \rightarrow H^{1}\left(S, \mathcal{O}_{S}\right) \rightarrow H^{1}\left(C, \mathcal{O}_{D}\right) \rightarrow \ldots
$$

one has $h^{1}\left(S, \mathcal{O}_{S}(-D)\right)>0$.

Assume in general that $h^{1}\left(S, \mathcal{O}_{S}(-D)\right)>0$. Then the intersection matrix $\left(D_{i} \cdot D_{j}\right)$ of the irreducible components $D_{1}, \ldots, D_{h}$ of $D$ is negative semi-definite. If it is not negative definite, then $D^{2}=0$ and there is an irrational pencil $f: S \rightarrow B$ such that a multiple of $D$ is a curve of the pencil.

If $h^{1}\left(S, \mathcal{O}_{S}(-D)\right)=q>0$, then the Albanese morphism of $S$ contracts $D$ to a point. If in addition $D^{2}=0$, then $B$ as above is the image of the Albanese map, and as such it is a smooth curve of genus $b=q$.

(0.10) (Ciliberto-Francia-Mendes Lopes, [CFM], 1.5-1.6). (a) Let $\mathcal{L}$ and $\mathcal{N}$ be line bundles on $S$. Assume that the general curve $C$ of $|\mathcal{L}|$ is irreducible, that the restriction of $\mathcal{N}$ to $C$ is trivial, and that either

(i) the general curve $C$ of $|\mathcal{L}|$ sits in a pencil $\mathcal{P}$ with at least a simple base point, and moreover $\mathcal{N}$ is numerically trivial on $S$; or

(ii) the general curve $C$ of $|\mathcal{L}|$ sits in a pencil $\mathcal{P}$ with a base point and no reducible curves.

Then $\mathcal{N}$ is trivial.

(b) Let $S$ be a surface, let $\mu \in \operatorname{Pic}^{0}(S)$ and let $C$ be a curve on $S$ such that $C^{2}>0$. Then $\mu$ is trivial if and only if $\mu_{\mid C}$ is trivial.

(0.11) (Ciliberto-Francia-Mendes Lopes, [CFM], A.4). Let $D$ be an m-connected curve on a surface $S$ and let $D=D_{1}+D_{2}$ with $D_{1}, D_{2}$ curves. If $D_{1} \cdot D_{2}=m$, then $D_{1}$ and $D_{2}$ are $[(m+1) / 2]$-connected.

(0.12) (Mendes Lopes, $[\mathrm{M}]$ ). Let $S$ be a surface and let $D$ be a curve on $S$. Then:

(a) If $D$ is 1-connected, a multiple point $x$ of $D$ is a base point of $|K+D|$ if and only if $D$ decomposes as a sum of two curves $A, B$ satisfying:

(i) $A \cdot B=1$;

(ii) $x$ is a non-singular point of $A$ and $\mathcal{O}_{A}(x) \simeq \mathcal{O}_{A}(B)$;

(iii) either $A \cap B=\{x\}$ or $A \leq B$.

(b) If $D$ is 2-connected, two distinct multiple points $x$ and $y$ of $D$ are not separated by $|K+D|$ if and only if $D$ decomposes as a sum of two curves $A, B$ satisfying:

(i) $A \cdot B=2$;

(ii) $x, y$ are non-singular points of $A$ and $\mathcal{O}_{A}(x+y) \simeq \mathcal{O}_{A}(B)$;

(iii) either $A \cap B=\{x, y\}$ or $A \leq B$.

(c) Suppose that $D$ is 2-connected and that $x$ is a point of $D$ such that mult $_{D}(x) \geq$ 3. Let $r$ be the rank of the differential of $\varphi_{K_{S}+D}$ at $x$.

If $r=1$, then $D$ has a decomposition $D=A+B$, where $A, B$ are curves such that $A \cdot B=2, A \cap B=\{x\}$ or $A \leq B$ and $x$ is either a non-singular point of $A$ or a double point of $A$.

If $r=0$, then $D$ has a decomposition $D=A_{1}+A_{2}+A_{3}$, where $A_{1}, A_{2}, A_{3}$ are curves such that:

(i) $A_{1} \cdot A_{2}=A_{1} \cdot A_{3}=A_{2} \cdot A_{3}=1$;

(ii) $\mathcal{O}_{A_{1}}(2 x) \simeq \mathcal{O}_{A_{1}}\left(A_{2}+A_{3}\right)$ and $\mathcal{O}_{A_{2}}(x) \simeq \mathcal{O}_{A_{2}}\left(A_{3}\right)$;

(iii) $x$ is a non-singular point of $A_{1}, A_{2}$; 
(iv) either $A_{1} \cap\left(A_{2}+A_{3}\right)=\{x\}$ or $A_{1} \leq\left(A_{2}+A_{3}\right)$, and either $A_{2} \cap A_{3}=\{x\}$ or $A_{2} \leq A_{3}$.

We notice that property $(i v)$ of the decomposition in part $(c)$ of the statement above is not contained in the statement of theorem (3.3) of [M], but its proof easily follows from the same arguments used in that paper.

(0.13) (Beltrametti-Sommese [BS]). Let $L$ be a nef divisor on a surface $S$. Assume that $L^{2} \geq 4 k+1$. Given any 0-dimensional scheme $Z$ of length $k$ on $S$, then either the natural restriction map

$$
H^{0}\left(S, \mathcal{O}_{S}(K+L)\right) \rightarrow H^{0}\left(S, \mathcal{O}_{Z}(K+L)\right)
$$

is surjective, or there exist an effective divisor $D$ on $S$ and a nonempty subscheme $Z^{\prime}$ of $Z$ of length $k^{\prime} \leq k$, such that:

(i) the map

$$
H^{0}\left(S, \mathcal{O}_{S}(K+L)\right) \rightarrow H^{0}\left(S, \mathcal{O}_{Z^{\prime}}(K+L)\right)
$$

is not surjective;

(ii) $Z^{\prime}$ is contained in $D$ and there is an integer $m$ such that $m(L-2 D)$ is effective;

(iii) one has

$$
L \cdot D-k^{\prime} \leq D^{2}<\frac{L \cdot D}{2}<k^{\prime}
$$

If $k=1$ then either $L \cdot D=0$ and $D^{2}=-1$, or $L \cdot D=1$ and $D^{2}=0$, and in either case $D$ is 1-connected.

Proof. For the proof we refer to [BS]. Only the statement concerning the 1connectedness of $D$ in the case $k=1$ needs some further explanation. Suppose that $L \cdot D=0$ and $D^{2}=-1$. If $D=A+B$, with curves $A$ and $B$, one has $L \cdot A=L \cdot B=0$ since $L$ is nef. Then the index theorem yields $A^{2}<0$ and $B^{2}<0$. This implies $-1=D^{2}=A^{2}+2 A \cdot B+B^{2} \leq 2 A \cdot B-2$, i.e. $A \cdot B>0$. The proof is similar in the case $L \cdot D=1$ and $D^{2}=0$. $\diamond$

We will now prove a few results concerning families of curves on a surface, which we will need later. They are certainly known to the experts, but we prove them here for lack of references.

Let $S$ be a smooth projective surface and let $T$ be a connected scheme. Recall that a family of curves on $S$ parametrized by $T$ is an effective relative Cartier divisor $\mathcal{C} \subset T \times S$. We will refer to the family $\mathcal{C} \rightarrow T$ if we want to put into evidence the parameter space $T$.

Let $t \in T$ be any closed point and let $\Gamma_{t}$ be the fibre of $p r_{1}$ over $t$. Then $C_{t}:=\operatorname{pr}_{2}\left(\Gamma_{t}\right)$ is a Cartier divisor on $S$ which is called the curve of the family $\mathcal{C}$ parametrized by $t$. Notice that $p_{a}\left(C_{t}\right)$ and the numerical equivalence class of $C_{t}$ are independent on $t \in T$. If $T$ is irreducible and $t$ is a general point of $T$, we will say that $C_{t}$ is a general curve of $\mathcal{C}$.

Given a family $\mathcal{C} \rightarrow T$ of curves on $S$, we can consider the biggest subdivisor $\mathcal{F}$ of $\mathcal{C}$ not dominating $S$ via the second projection $p r_{2}: \mathcal{C} \rightarrow S$. The family $\mathcal{F}$ is constant, i.e. the curve $F:=F_{t}$ is independent of $t \in T$, and it is called the fixed part of $\mathcal{C}$, inasmuch as $F$ is contained in any curve $C_{t}$ of the family $\mathcal{C}$.

Given a family $\mathcal{C} \rightarrow T$ as above, and given any point $x \in S$, we can consider the locus $T_{x}:=\left\{t \in T: x \in C_{t}\right\}$, which has an obvious structure of closed subscheme of 
$T$. The family $\mathcal{C}_{x} \rightarrow T_{x}$ induced by $\mathcal{C}$ on $T_{x}$ is the family of all curves in $\mathcal{C}$ passing through $x$. Notice that if $T$ is irreducible and $x$ is a general point of $S$, then any irreducible component of $T_{x}$ has codimension one in $T$.

As a particular case, we will consider the situation in which $T$ is a subscheme of the Hilbert scheme of curves of given arithmetic genus $g$ on $S$, and $\mathcal{C} \rightarrow T$ is induced by the universal family. We will always consider the case in which $T$ is a closed, reduced subscheme of the Hilbert scheme. In this case we will say that $\mathcal{C} \rightarrow T$ is a system of curves on $S$. If $T$ is irreducible of dimension $r$, we will say that the system of curves is irreducible of dimension $r$. Moreover we will say that the system is complete if $T$ is an irreducible component of the Hilbert scheme. If $S$ is a regular surface, then complete irreducible systems on $S$ coincide with complete linear systems.

The $r$-dimensional system $\mathcal{C} \rightarrow T$ is said to be composed with the $r_{i}$-dimensional systems $\mathcal{C}_{i}, i=1, \ldots, h$, if the general, or equivalently any, curve $C$ of $\mathcal{C}$ can be expressed as $C=C_{1}+\ldots+C_{h}$, with $C_{i}$ a curve of $\mathcal{C}_{i}, i=1, \ldots, h$. Notice that $r \leq r_{1}+\ldots+r_{h}$ and the equality holds if and only if the general curve $C$ of $\mathcal{C}$ can be expressed as $C=C_{1}+\ldots+C_{h}$, with $C_{i}$ the general curve of $\mathcal{C}_{i}, i=1, \ldots, h$. If in the above setting the curves $C_{i}$ of $\mathcal{C}_{i}$ are the irreducible components of $C$, we will say that $\mathcal{C}$ is irreducibly composed with the systems $\mathcal{C}_{i}, i=1, \ldots, h$. If in addition one has $r=r_{1}+\ldots+r_{h}$, then we will say that $\mathcal{C}$ is completely irreducibly composed with the systems $\mathcal{C}_{i}, i=1, \ldots, h$. If $\mathcal{C}_{i}=\mathcal{D}, i=1, \ldots, h$, one says that $\mathcal{C}$ is composed with the system $\mathcal{D}$. In particular if $\mathcal{D}$ is a base point free pencil, i.e. it is formed by the fibres of a surjective morphism $f: S \rightarrow B$ of $S$ to a curve $B$, then any curve $C$ of $\mathcal{C}$ is such that $C^{2}=0$.

We are going to list in the following lemma a few basic properties of irreducible systems which have no fixed part. Before stating the lemma, we make a definition. Let $B_{1}, B_{2}$ be smooth irreducible projective curves, and consider the product $B_{1} \times$ $B_{2}$. The two projection morphisms $p_{i}: B_{1} \times B_{2} \rightarrow B_{i}$ give rise to two base point free pencils $\mathcal{P}_{i}, i=1,2$, on $B_{1} \times B_{2}$. We call the curves of $\mathcal{P}_{i}$ the $i$-th coordinate curves of $B_{1} \times B_{2}$. We also notice the 2-dimensional system $\mathcal{P}$ of curves on $B_{1} \times B_{2}$ which is completely irreducibly composed with the systems $\mathcal{P}_{i}, i=1,2$. We will call $\mathcal{P}$ the system of pairs of coordinate curves of $B_{1} \times B_{2}$. We have:

(0.14) Lemma. Let $\mathcal{C} \rightarrow T$ be an irreducible $r$-dimensional system of curves on a surface $S$ and let $C$ be the general curve of $\mathcal{C}$. Suppose that $\mathcal{C}$ has no fixed part; in particular, $r \geq 1$. Then:

(i) one has $C^{2}=0$ if and only if $\mathcal{C}$ is composed with a pencil without base points;

(ii) if $\mathcal{C}$ is complete, if $x$ is a general point of $S$ and if the subsystems of $\mathcal{C}_{x} \rightarrow T_{x}$ induced on the irreducible components of $T_{x}$ all have a fixed part, then either $r=1$ or $r \geq 2$ and $\mathcal{C}$ is completely irreducibly composed with $r$ irreducible 1-dimensional systems $\mathcal{C}_{i}, i=1, \ldots, r$

(iii) in case (ii) one has either $C^{2}=0$ or $C^{2} \geq r$. Moreover $C^{2}=r \geq 2$ if and only if $r=2$ and $\mathcal{C}_{i}, i=1,2$, are base point free pencils such that $C_{1} \cdot C_{2}=1$ for $C_{i}$ the general curve in $\mathcal{C}_{i}, i=1,2$. Then there is a birational morphism $f: S \rightarrow B_{1} \times B_{2}$, where $B_{1}, B_{2}$ are smooth irreducible projective curves, and $f$ maps $\mathcal{C}$ to the family $\mathcal{P}$ of pairs of coordinate curves on $B_{1} \times B_{2}$.

Proof. Let $C$ be the general curve of $\mathcal{C}$. We write $C=C_{1}+\ldots+C_{h}, h \geq 1$, where $C_{1}, \ldots, C_{h}$ are the irreducible components of $C$. They all move, since $\mathcal{C}$ has no fixed 
component; therefore all of them are nef. Then

$$
C^{2}=\sum_{i=1}^{h} C_{i}^{2}+2 \sum_{1 \leq i<j \leq h}^{h} C_{i} \cdot C_{j}
$$

Hence $C^{2} \geq 0$, and $C^{2}=0$ if and only if $C_{i} \cdot C_{j}=0$ for all $1 \leq i \leq j \leq h$. So $C^{2}=0$ implies that all components of $C$ describe, as $C$ moves in $\mathcal{C}$, one and the same base point free pencil, and $\mathcal{C}$ is composed with it. We have thus proved (i).

In order to prove (ii) we start by remarking that if $r \geq 2$, if the general curve $C$ of $\mathcal{C}$ is irreducible, and if $x$ is a general point of $S$, then the subsystems of $\mathcal{C}_{x} \rightarrow T_{x}$ induced on the irreducible components of $T_{x}$ all have no fixed part. In fact, any such irreducible component has dimension $r-1 \geq 1$ and parametrizes the general curve of $\mathcal{C}$, which is irreducible.

Again let $C=C_{1}+\ldots+C_{h}, h \geq 1$, be the general curve of $\mathcal{C}$, where $C_{1}, \ldots, C_{h}$ are its irreducible components. As $C$ describes $\mathcal{C}$, the curve $C_{i}$ also varies, describing an irreducible system contained in some complete system $\mathcal{C}_{i}$ of dimension $r_{i}, i=$ $1, \ldots, h$. Since $\mathcal{C}$ has no fixed part, we have $r_{i}>0, i=1, \ldots, h$. Since $\mathcal{C}$ is complete, it is completely irreducibly composed with the systems $\mathcal{C}_{i}, i=1, \ldots, h$.

If $r_{i}>1$ for some $i=1, \ldots, h$, then for $x$ general in $S$, all irreducible components of $\mathcal{C}_{i, x}$ have no fixed part. This clearly yields the existence of irreducible components of $\mathcal{C}_{x}$ with no fixed part. Hence if all irreducible components of $\mathcal{C}_{x}$ have a fixed part, we must have $r_{i}=1, i=1, \ldots, h$, and therefore $h=r$ since $r=r_{1}+\ldots+r_{h}$. We have thus proved (ii).

To prove (iii), we notice that $C \cdot C_{i} \geq 0$ for all $i=1, \ldots, r$, since the $C_{i}$ 's are nef. If for some $i=1, \ldots, r$ one has $C \cdot C_{i}=0$, then $C_{i} \cdot C_{j}=0$, for all $j=1, \ldots, r$. Then $\mathcal{C}_{i}$ is a base point free pencil and $\mathcal{C}$ is composed with it, which implies $C^{2}=0$. Otherwise we have $C \cdot C_{i}>0$ for all $i=1, \ldots, h$, which yields $C^{2} \geq r$. Assume $C^{2}=r \geq 2$. This means that $C \cdot C_{i}=1$ for all $i=1, \ldots, r$. We claim that $C_{i}^{2}=0$ for all $i=1, \ldots, r$. Otherwise we have an $i \in\{1, \ldots, r\}$ such that $C_{i}^{2}=1$ and $C_{i} \cdot C_{j}=0$, for all $j=1, \ldots, r, j \neq i$, contradicting the index theorem. Furthermore, for any $i=1, \ldots, r$, there is a unique $j \in\{1, \ldots, r\}, j \neq i$, such that $C_{i} \cdot C_{j}=1$, whereas $C_{i} \cdot C_{k}=0$ for all $k=1, \ldots, r, j \neq k$. This clearly forces $r=2$ and $C=C_{1}+C_{2}$, with $C_{i}^{2}=0, C_{1} \cdot C_{2}=1$. Then $\mathcal{C}_{i}, i=1,2$, are base point free pencils $f_{i}: S \rightarrow B_{i}$, with $B_{i}$ smooth projective curves. The map $f=f_{1} \times f_{2}: S \rightarrow B_{1} \times B_{2}$ is the required birational morphism. $\diamond$

Now we have the following:

(0.16) Proposition. Let $\mathcal{C} \rightarrow T$ be an irreducible $r$-dimensional family of curves on a surface $S$, with $r \geq 1$, and let $C$ be the general curve in $\mathcal{C}$. If $\mathcal{C}$ has no fixed part, then:

(i) either $C^{2}=0$ or $C^{2} \geq r-1$;

(ii) one has $C^{2}=r-1$, with $r \geq 2$, if and only if $\mathcal{C}$ is a complete, base point free, linear system $|C|$ of curves of arithmetic genus zero such that the morphism $\phi_{|C|}: S \rightarrow \Sigma \subseteq \mathbf{P}^{r}$ is birational to its image $\Sigma$, which is a rational normal surface of degree $r-1$.

Proof. Since $C$ is nef, we have $C^{2} \geq 0$. Let us assume $C^{2}>0$. Assertion (i) is trivial if $r=1$, and assertion (ii) is empty in that case. Hence we may assume $C^{2} \geq 1$ and $r \geq 2$. If $r=2$ then (i) is clear again. If $C^{2}=1$, we have that $C$ is 
irreducible; otherwise by (0.15) we would have $C_{i} \cdot C_{j}=0$, for all $1 \leq i<j \leq h$ and, say, $C_{1}^{2}=1, C_{i}^{2}=0, i=2, \ldots, h$. This would contradict the index theorem.

Since $H^{0}\left(C, \mathcal{O}_{C}(C)\right)$ is the tangent space to the Hilbert scheme of curves on $S$ at the point representing $C$, and the Hilbert scheme, containing $\mathcal{C}$, has dimension at least 2, one has $h^{0}\left(C, \mathcal{O}_{C}(C)\right) \geq 2$. Since $C^{2}=1$, we have that $h^{0}\left(C, \mathcal{O}_{C}(C)\right)=2$ and $\mathcal{C}$ is complete. Moreover $C$ is rational and $S$, having a 2-dimensional system of rational curves with positive self-intersection, is rational. By looking at the exact sequence

$$
\begin{gathered}
0 \rightarrow H^{0}\left(S, \mathcal{O}_{S}\right) \rightarrow H^{0}\left(S, \mathcal{O}_{S}(C)\right) \rightarrow H^{0}\left(C, \mathcal{O}_{C}(C)\right) \\
\rightarrow H^{1}\left(S, \mathcal{O}_{S}\right) \rightarrow H^{1}\left(S, \mathcal{O}_{S}(C)\right) \rightarrow \ldots
\end{gathered}
$$

we see that $|C|$ is base point free of dimension 2; hence $|C|$ coincides with $\mathcal{C}$ and (ii) easily follows in this case.

Let us now assume $r \geq 3$ and argue by induction to prove (i) and (ii). Let $x$ be a general point of $S$. By lemma $(0.14$, iii), we may assume that there is an irreducible component $T_{x}^{(1)}$ of $T_{x}$ such that the induced system $\mathcal{C}_{x}^{(1)} \rightarrow T_{x}^{(1)}$ has no fixed part. Let $\mu \geq 1$ be the multiplicity at $x$ of the general curve of $\mathcal{C}_{x}^{(1)}$. Let $p: S^{\prime} \rightarrow S$ be the blow-up of $S$ at $x$ and let $E$ be the exceptional divisor. Let us consider the strict transform $\mathcal{C}^{\prime} \rightarrow T_{x}^{(1)}$ of $\mathcal{C}_{x}^{(1)} \rightarrow T_{x}^{(1)}$ on $S^{\prime}$, i.e. $\mathcal{C}^{\prime}$ is the system whose general curve is given by $C^{\prime}=p^{*}(C)-\mu E$, where $C$ is the general curve in $\mathcal{C}_{x}^{(1)}$. We have that $\mathcal{C}^{\prime}$ is irreducible, of dimension $r-1 \geq 2$, and without fixed part, since $\mathcal{C}_{x}^{(1)}$ has no fixed part and $E$ also does not appear in its fixed locus. By induction we have either $C^{2}=0$ or $C^{2} \geq r-2$.

Suppose that $C^{\prime 2}=0$. Then $\mathcal{C}^{\prime}$ is composed with a pencil $\mathcal{D}$ whose general curve we denote by $D$. Hence the general curve $C^{\prime}$ of $\mathcal{C}^{\prime}$ is of the form $C^{\prime}=$ $D_{1}+\ldots+D_{k}$, with $D_{i}, i=1, \ldots, k$, its irreducible components, curves of $\mathcal{D}$. Since $0<\mu=C^{\prime} \cdot E=k D \cdot E$, we have $D \cdot E>0$. Since $\mathcal{C}^{\prime}$ has dimension $r-1$ we have $k \geq r-1$; hence $\mu=k D \cdot E \geq r-1$. On the other hand we have $C^{2}=p^{*}(C)^{2}=(k D+\mu E)^{2}=2 k \mu D \cdot E-\mu^{2}=\mu^{2} \geq(r-1)^{2}>r-1$. If instead $C^{\prime 2} \geq r-2$, then $r-2 \leq C^{\prime 2}=C^{2}-\mu^{2}$, which yields $C^{2} \geq r-1$. We have thus proved (i).

Assume now that $C^{2}=r-1$, which in our setting yields $C^{2}=r-2$ and $\mu=1$. By induction, $\mathcal{C}^{\prime}$ is a complete, base point free linear system of curves of arithmetic genus zero on $S^{\prime}$, which is rational. So also $S$ is rational. The general curve $C^{\prime}$ of $\mathcal{C}^{\prime}$ is smooth, irreducible and rational, which implies that also the general curve $C$ of $\mathcal{C}$ is smooth, irreducible and rational. By looking at the sequence $(0.17)$ we see that $\mathcal{C}$ is a complete, base point free linear system $|C|$ of curves of arithmetic genus zero on $S$. Finally, the last assertion about the map determined by $|C|$ is clear. $\diamond$

Next we are going to characterize the systems $\mathcal{C}$ as above for which $C^{2}=r$.

First we recall another definition. Given an irreducible 1-dimensional family $\mathcal{C} \rightarrow T$ of curves on a surface $S$, the index $\nu:=\nu_{\mathcal{C}}$ of $\mathcal{C}$ is the degree of the second projection $p r_{2}: \mathcal{C} \rightarrow S$, or, equivalently, the number of curves of $\mathcal{C}$ passing through the general point of $S$. Notice that $\nu=0$ if and only if $p r_{2}: \mathcal{C} \rightarrow S$ is not dominant, which never occurs if we consider a system. Moreover, $\nu=1$ if and only if $\mathcal{C}$ is birational to $S$, in which case there is a rational map $f: S \rightarrow T$, i.e. a pencil, and $\mathcal{C}$ is clearly composed with the system of the fibres of $f$. 
Let $C$ be a smooth projective curve and let $C^{(2)}$ be its symmetric product, i.e. the Hilbert scheme of divisors of degree two on $C$. We denote by $C_{x}$ the curve on $C^{(2)}$ described by all divisors of the form $x+y$, when $y$ varies in $C$. This is called a coordinate curve on $C^{(2)}$. The system of coordinate curves on $C^{(2)}$ is 1-dimensional, of index two, parametrized by $C$.

(0.18) Proposition. Let $\mathcal{C}$ be an irreducible 1-dimensional system of curves on a smooth projective surface $S$, without fixed part. If the general curve $C$ of $\mathcal{C}$ is such that $C^{2}=1$, then it is irreducible, smooth and:

(i) either $\mathcal{C}$ is a subsystem of a complete, base point free, linear system $|C|$ of dimension 2 such that the morphism $\phi_{|C|}: S \rightarrow \mathbf{P}^{2}$ is a sequence of blow-ups of the plane;

(ii) or $\mathcal{C}$ is a complete linear system with one transversal base point;

(iii) or $C$ has genus $g>0$ and there is a birational map $S \rightarrow C^{(2)}$ which sends $\mathcal{C}$ to the system of coordinate curves on $C^{(2)}$.

Proof. Let $C=C_{1}+\ldots+C_{h}$ be the general curve of $\mathcal{C}$, where $C_{1}, \ldots, C_{h}, h \geq 1$, are the irreducible components of $C$. Using (0.15) we see that $C^{2}=1$ and $h \geq 2$ contradict the index theorem. So $C$ is irreducible.

The section of $H^{0}\left(C, \mathcal{O}_{C}(C)\right)$ corresponding to the infinitesimal deformation of $C$ inside $\mathcal{C}$ is non-zero and it is well known to vanish at every singular point of $C$. Since $C$ is irreducible and $C^{2}=1$, then $C$ must be smooth.

Suppose $\mathcal{C}$ is a subsystem of a linear system $|C|$. If $|C|$ has a base point, we are in case (ii). Otherwise, by looking at the sequence (0.17), we see that $|C|$ is base point free of dimension 2 , its general curve $C$ is smooth and rational, and we are in case (i).

Suppose that $\mathcal{C}$ is not a subsystem of a linear system, and let us prove that $\nu=2$. We have $\nu \geq 2$, because $\nu=1$ and $\mathcal{C}$ not a subsystem of a linear system forces $\mathcal{C}$ to be an irrational pencil, and then $C^{2}=0$, a contradiction. Suppose that $\nu \geq 3$. Let $x$ be a general point of $S$ and let $C_{1}, \ldots, C_{\nu}$ be the curves of $\mathcal{C}$ through $x$. By $(0.10, \mathrm{~b})$ one deduces that, for all $i \in\{1, \ldots, \nu\}$, the curves $C_{1}, \ldots, C_{i-1}, C_{i+1}, \ldots, C_{\nu}$ are linearly equivalent. Since we are assuming $\nu \geq 3$, this yields that $C_{1}, \ldots, C_{\nu}$ are linearly equivalent, and this in turn implies that all curves in $\mathcal{C}$ are linearly equivalent, a contradiction.

Notice that $\mathcal{C}$ is base point free, as otherwise we would have $\nu=1$. Moreover $C$ has genus $g>0$, since we are assuming that $\mathcal{C}$ is not a subsystem of a larger linear system. Let $C, C^{\prime}$ be two general curves of $\mathcal{C}$. Let $x$ be a general point of $C$ and let $D$ be the curve in $\mathcal{C}$ different from $C$ through $x$. Let $y$ be the intersection point of $D$ with $C^{\prime}$. Hence we have a birational map $x \in C \rightarrow y \in C^{\prime}$, i.e. $C$ and $C^{\prime}$ are isomorphic. Finally, let $x$ be a general point in $S$ and let $C^{\prime}, C^{\prime \prime}$ be the two curves in $\mathcal{C}$ passing through $x$. Let $y$ and $z$ be the intersection points of $C^{\prime}$ and $C^{\prime \prime}$ with $C$. The map $x \in S \rightarrow y+z \in C^{(2)}$ is the required birational map. $\diamond$

Before proving our classification result for the systems $\mathcal{C}$ of dimension $r$ with $C^{2}=r$, we need one more lemma:

(0.19) Lemma. Let $S$ be a smooth projective surface and let $C$ be a smooth irreducible elliptic curve on $S$ with $C^{2}=r>0$. Then $S$ is birationally ruled, and $C$ belongs to a unique irreducible complete $r$-dimensional system $\mathcal{C}$ of curves on $S$ such that either:

(i) $\mathcal{C}$ is a linear system, or 
(ii) $q=1$ and $\mathcal{C}$ consists of a 1-dimensional system of complete linear systems of dimension $r-1$.

Proof. First of all, $K \cdot C=-r$ implies $S$ is birationally ruled. Since $h^{1}\left(C, \mathcal{O}_{C}(C)\right)$ $=0$, the Hilbert scheme of curves on $S$ is smooth of dimension $h^{0}\left(C, \mathcal{O}_{C}(C)\right)=r$ at $C$. Hence $C$ belongs to a unique complete system $\mathcal{C}$ of dimension $r$. Suppose that $\mathcal{C}$ is not a linear system, so that $h^{0}\left(S, \mathcal{O}_{S}(C)\right) \leq r$. By looking at the sequence (0.17) we see that $S$ must be irregular. But since $C^{2}>0$, the curve $C$ dominates the image of the Albanese map, and therefore we have $q=1$. The sequence $(0.17)$ yields that $|C|$ has dimension $r-1$, proving (ii). $\diamond$

Now we are ready to prove our classification theorem:

(0.20) Theorem. Let $\mathcal{C} \rightarrow T$ be an irreducible $r$-dimensional system of curves on a smooth projective surface $S$, without fixed part and such that the general curve $C$ of $\mathcal{C}$ has $C^{2}=r \geq 1$ and arithmetic genus $g$. Then $C$ is irreducible and smooth, and:

(i) either $\mathcal{C}$ is not complete, in which case $\mathcal{C}$ is a subsystem of a complete, base point free, linear system $|C|$ of dimension $r+1$ of curves with $g=0$ such that the morphism $\phi_{|C|}: S \rightarrow \Sigma \subseteq \mathbf{P}^{r+1}$ is a sequence of blow-ups of a rational normal scroll;

(ii) or $\mathcal{C}$ is complete, in which case either:

$\left(i i_{1}\right) \mathcal{C}$ is a linear system; or

$\left(i i_{2}\right) r=1$, the curve $C$ has genus $g>0$ and there is a birational map $S \rightarrow C^{(2)}$ which sends $\mathcal{C}$ to the system of coordinate curves on $C^{(2)}$; or

$\left(i i_{3}\right) r \geq 2$ and then either:

$\left(i i_{3,1}\right) g=r=2$ and there is a birational map $S \rightarrow A$, where $A \simeq \operatorname{Jac}(C)$ is birational to $C^{(2)}$, sending $\mathcal{C}$ to the system of theta divisors on $A$; or

$\left(i i_{3,2}\right) g=1$, the system $\mathcal{C}$ consists of a 1 -dimensional system of complete linear systems of dimension $r-1$ and there is a birational map $S \rightarrow C^{(2)}$; or

$\left(i i_{3,3}\right) r=2$ and there is a birational morphism $f: S \rightarrow B_{1} \times B_{2}$, where $B_{1}, B_{2}$ are smooth irreducible projective curves and $f$ maps $\mathcal{C}$ to the system $\mathcal{P}$ of pairs of coordinate curves on $B_{1} \times B_{2}$.

Proof. For $r=1$ the assertion follows by proposition (0.18). If $\mathcal{C}$ is not complete the assertion follows by proposition (0.16). So we may assume $r \geq 2$ and $\mathcal{C}$ complete.

Let $x$ be a general point of $S$. If the systems induced by $\mathcal{C}_{x} \rightarrow T_{x}$ on the irreducible components of $T_{x}$ all have a fixed part, we are in case $\left(i i_{3,3}\right)$ by lemma $\left(0.14\right.$, iii). So we may assume that there is an irreducible component $T_{x}^{(1)}$ of $T_{x}$ such that the induced system $\mathcal{C}_{x}^{(1)} \rightarrow T_{x}^{(1)}$ has no fixed part. Let $\mu \geq 1$ be the multiplicity of the general curve of $\mathcal{C}_{x}^{(1)}$ at $x$.

Let $p: S^{\prime} \rightarrow S$ be the blow-up of $x$ and let $E$ be the exceptional divisor. Let $\mathcal{C}^{\prime}$ be the strict transform of $\mathcal{C}_{x}^{(1)}$ via $p$. The general curve $C^{\prime}$ of $\mathcal{C}^{\prime}$ is $C^{\prime}=p^{*}(C)-\mu E$, where $C$ is the general curve in $\mathcal{C}_{x}^{(1)}$. If $C^{\prime 2}=0$ then, as we saw in the proof of proposition (0.16), we have $C^{2} \geq(r-1)^{2}$. When $C^{2}=r$ we must have $r=2$. On the other hand we have $0=C^{2}=C^{2}-\mu^{2}=2-\mu^{2}$, a contradiction.

So we have $r-2 \leq C^{\prime 2}=C^{2}-\mu^{2}=r-\mu^{2}$ by proposition (0.16). Hence $\mu=1$ and $C^{\prime 2}=r-1$. By induction $C^{\prime}$ is smooth and irreducible, hence so is $C$.

Suppose $\mathcal{C}^{\prime}$ is a subsystem of a linear system. Then all curves in $\mathcal{C}_{x}^{(1)}$ are linearly equivalent. As $x$ varies on $S$, the family $\mathcal{C}_{x}^{(1)}$, which has no fixed part, varies in $\mathcal{C}$ 
describing a 2-dimensional family of irreducible subvarieties of codimension one. A general curve $C$ in $\mathcal{C}$ belongs to a one-dimensional family of systems of the type $\mathcal{C}_{x}^{(1)}$, which sweep out the whole of $\mathcal{C}$. Hence all curves of $\mathcal{C}$ are linearly equivalent and we are in case $\left(i i_{1}\right)$.

So we may assume that $\mathcal{C}^{\prime}$ is not a subsystem of a linear system, so $\mathcal{C}$ is not a linear system. Take again a general curve $C$ of $\mathcal{C}$. By Hilbert scheme considerations, we have $h^{0}\left(C, \mathcal{O}_{C}(C)\right) \geq r$. If $h^{0}\left(C, \mathcal{O}_{C}(C)\right) \geq r+1$, then $C$ would be rational and $\mathcal{C}$ and $\mathcal{C}^{\prime}$ would be subsystems of linear systems, a contradiction. Thus we have $h^{0}\left(C, \mathcal{O}_{C}(C)\right)=r$. Since $r \geq 2$, we have either $r=2$ and $C$ is hyperelliptic, or $g=1$.

If $g=1$ the first part of $\left(i i_{3,2}\right)$ follows by lemma (0.19). As for the second part, it is immediately proved by induction, since it holds for $r=1$ and the induction can be applied to $\mathcal{C}^{\prime}$, proving that $S^{\prime}$, and therefore $S$, is birational to $C^{(2)}$.

Finally let us assume $r=2$ and $g \geq 2$. By proposition (0.18) we have a birational map $f: S^{\prime} \rightarrow C^{(2)}$ sending $\mathcal{C}^{\prime}$ to the family of coordinate curves on $C^{(2)}$. The map $f$ cannot contract the exceptional divisor $E$, as otherwise the self-intersection of the images via $f$ of the curves of $\mathcal{C}^{\prime}$ would be at least 2 . Hence $E$ is sent to a curve $R$ representing all the divisors of degree 2 in a $g_{2}^{1}$ on $C$. For such a curve one has $R^{2}=1-g$. Notice that $f$ has no fundamental point on $E$; otherwise $C^{(2)}$ would contain some other rational curve besides $R$, which is impossible because there is no more than one $g_{2}^{1}$ on $C$. Hence we have $R^{2}=-1$; thus $g=2$. Furthermore, $R$ is also an exceptional curve on $C^{(2)}$ and, by contracting it, we have the Abel-Jacobi morphism $C^{(2)} \rightarrow A \simeq \operatorname{Jac}(C)$. The existence of the birational map $S^{\prime} \rightarrow C^{(2)}$ yields the existence of a birational map $S \rightarrow A \simeq \operatorname{Jac}(C)$ which sends the general curve $C$ of $\mathcal{C}$ to the Abel-Jacobi image of $C$ in $A$. This proves that we are in case $\left(i i_{3,1}\right) . \diamond$

We finish this section with the following very simple lemma:

(0.21) Lemma. Let $S$ be a smooth projective surface and let $f: S \rightarrow B$ be an irrational pencil on $S$. Let $\Gamma$ be an irreducible curve on $S$ of geometric genus $g$, not contained in any curve of the pencil and passing through distinct points $x_{1}, \ldots, x_{h}$ having respective multiplicities $m_{1}, \ldots, m_{h}$ for the fibres of the pencil. Then $2 g \geq 2+\sum_{i=1}^{h}\left(m_{i}-1\right)$. In particular, if the irrational pencil contains some multiple fibre, then all elliptic and rational curves on $S$ are contained in curves of the pencil.

Proof. Let $C$ be the normalization of $\Gamma$. The morphism $f$ induces a morphism $\phi: C \rightarrow B$ of positive degree. The assertion follows by applying Hurwitz's formula to $\phi$ and by taking into account that $b \geq 1$. $\diamond$

\section{The CASE $K^{2}=9$}

In [R] I. Reider proved that if $S$ is a minimal surface of general type with $K^{2} \geq 10$ and the bicanonical map is not birational, then $S$ presents the standard case. He also remarks that the same holds even if $K^{2}=9$ unless the canonical system is numerically divisible by three. We can make this remark more precise in the case $p_{g} \geq 3$. Recall that a reduced curve $C$ on a surface $S$ has non-essential singularities if it has only A-D-E singularities (see [BPV], pg. 87). We have the following:

(1.1) Proposition. Let $S$ be a minimal surface of general type with $K^{2}=9$ and $p_{g} \geq 3$ such that the bicanonical map is not birational. Assume that $S$ presents the 
non-standard case. Then $p_{g}=6, q=0$, and $S$ is the minimal model of a double cover $\varphi: S^{\prime} \rightarrow \mathbf{F}_{2}=\operatorname{Proj}\left(\mathcal{O}_{\mathbf{P}^{1}} \oplus \mathcal{O}_{\mathbf{P}^{1}}(-2)\right)$ with branch curve $B$ sitting in the linear system $\left|14 F+8 C_{0}\right|$, where $F$ is a fibre and $C_{0}$ is the (-2)-section of $\mathbf{F}_{2}$. Moreover the curve $B$ breaks up as $C_{0}+B^{\prime}$, where $B^{\prime}$ is such that:

(i) $B^{\prime} \cap C_{0}=\emptyset$;

(ii) $B^{\prime}$ is reduced and has at most non-essential singularities.

Proof. Let $x, y$ be two general points of $S$ such that $\phi(x)=\phi(y)$. By $(0.13)$ there is an effective divisor $D$ on $S$ such that $x, y \in D$ and, moreover,

$$
-4 \leq 2 K \cdot D-4 \leq 2 D^{2} \leq K \cdot D \leq 3 \text {. }
$$

Therefore $1 \geq D^{2} \geq-2$. As $x$ and $y$ vary, the divisor $D$ also varies, describing an irreducible system $\mathcal{D}$ of curves on $S$, which has a movable part $M$ and a fixed part $F$.

Claim 1. $F=0, D^{2}=1$ and $K \sim 3 D$; moreover the general curve $D$ of $\mathcal{D}$ is smooth and irreducible. Of course $M^{2} \geq 0$. One cannot have $M^{2}=0$. In fact (1.2) yields $3 \geq K \cdot D \geq K \cdot M>0$, and therefore we would have $K \cdot M=2$, i.e. $S$ would present the standard case. Hence $M^{2}>0$, which implies, by the index theorem, $M^{2}=1$ and $K \sim 3 M$. Since $3=K \cdot M \leq K \cdot D \leq 3$, we have also $K \cdot D=3$ and $D^{2}=1$ by (1.2). The index theorem again implies $K \sim 3 D$; hence $F \sim 0$, i.e. $F=0$. The rest of the assertion follows by proposition $(0.18)$.

Claim 2. $D$ is 2-connected. Since $K \sim 3 D$, the divisor $D$ is nef. Moreover by (0.11) it is also 1-connected, since $K$ is 2-connected. Suppose that $D=A+B$ with $A, B$ effective and such that $A \cdot B=1$. Since $1=D^{2}=D \cdot A+D \cdot B$, then, say, $D \cdot A=1$ and $D \cdot B=0$; hence $A^{2}=0$. But since $K \cdot A=3 D \cdot A=3$, we have a contradiction.

Claim 3. The family $\mathcal{D}$ is a linear system of dimension 1 with a transversal base point $p$, and the bicanonical map $\phi$ is non birational on the curves of $\mathcal{D}$. The first assertion follows by proposition (0.18). In fact the only other possibility would be that $S$ is isomorphic to the symmetric product of a smooth curve of genus $g$, which is known to have $p_{g}=\frac{g(g-1)}{2}, K^{2}=4 g^{2}-13 g+9$, and this is incompatible with our hypotheses $p_{g} \geq 3$ and $K^{2}=9$. The second assertion follows from the first and the fact that pairs of points $(x, y) \in S \times S$ such that $\phi(x)=\phi(y)$ lie on curves of $\mathcal{D}$.

Claim 4. Any irreducible curve $D \in \mathcal{D}$ is hyperelliptic. Let us consider the exact sequence

$$
0 \rightarrow \mathcal{O}_{S}(K-D) \rightarrow \mathcal{O}_{S}(K) \rightarrow \mathcal{O}_{D}(K) \rightarrow 0 .
$$

Since $O_{D}(K)$ has degree 3 and $D$ has arithmetic genus 3 , one has $h^{0}\left(D, O_{D}(K)\right) \leq$ 2. Therefore $h^{0}\left(S, O_{S}(K-D)\right)>0$. Consider now the exact sequence

$$
0 \rightarrow \mathcal{O}_{S}(2 K-D) \rightarrow \mathcal{O}_{S}(2 K) \rightarrow \mathcal{O}_{D}(2 K) \rightarrow 0 .
$$

Since $h^{0}\left(S, O_{S}(K-D)\right)>0$, there are effective divisors in $|K-D|$. Since $K-D \sim$ $2 D$, any divisor $C$ in $|K-D|$ is 1-connected by (0.11) applied to the decomposition $K=C+D$. Furthermore one has $(K-D)^{2}>0$. Hence $h^{1}\left(S, O_{S}(2 K-D)\right)=0$, and therefore the map $H^{0}\left(S, O_{S}(2 K)\right) \rightarrow H^{0}\left(D, O_{D}(2 K)\right)$ is surjective. Since the degree of $O_{D}(2 K)$ is 6 and $D$ has arithmetic genus 3, by claim 3 we have the assertion.

Claim 5. Let $D$ be the general curve in $\mathcal{D}$. The base point $x$ of $\mathcal{D}$ is a Weierstrass point on $D$. Furthermore $K \equiv 3 D$. Let $\eta$ be the hyperelliptic line bundle on $D$. 
Since $O_{D}(2 K) \equiv \eta^{\otimes 3}$ and $O_{D}(K+D) \equiv \eta^{\otimes 2}$, the base point $x$ of $\mathcal{D}$ is a Weierstrass point. Now $\mathcal{O}_{D}(K+D) \simeq \eta^{\otimes 2} \simeq \mathcal{O}_{D}(4 D)$, and the conclusion follows by $(0.10$, a).

Let $\sigma: S^{\prime} \rightarrow S$ be the blow-up of $S$ at $x$ and let $E$ be the exceptional divisor. We denote by $p: S^{\prime} \rightarrow \mathbf{P}^{1}$ the morphism determined by the pull-back on $S^{\prime}$ of the pencil $\mathcal{D}$. Since every curve $C \in \mathcal{D}$ is 2 -connected by claim 2 and $h^{0}\left(C, \mathcal{O}_{C}(2 x)\right) \geq 2$ by semicontinuity, we have $h^{0}\left(C, \mathcal{O}_{C}(2 x)\right)=2$ (see proposition (A.5) of the Appendix of $[\mathrm{CFM}])$. So the sheaf $p_{*}\left(\mathcal{O}_{S^{\prime}}(2 E)\right)$ is a locally free sheaf of rank 2. Moreover we have $h^{0}\left(\mathbf{P}^{1}, p_{*}\left(\mathcal{O}_{S^{\prime}}(2 E)\right)\right)=1$; hence $p_{*}\left(\mathcal{O}_{S^{\prime}}(2 E)\right) \simeq \mathcal{O}_{\mathbf{P}^{1}} \oplus \mathcal{O}_{\mathbf{P}^{1}}(-e)$, where $e$ is a positive integer. As usual, we set $\mathbf{F}_{e}=\operatorname{Proj}\left(\mathcal{O}_{\mathbf{P}^{1}} \oplus \mathcal{O}_{\mathbf{P}^{1}}(-e)\right)$; we denote by $F$ a fibre of the structure map $\mathbf{F}_{e} \rightarrow \mathbf{P}^{1}$, and by $C_{0}$ the curve of $\mathbf{F}_{e}$ such that $C_{0}^{2}=-e$.

For every curve $C \in \mathcal{P}$ the sheaf $\mathcal{O}_{C}(2 x)$ is generated by global sections (see proposition (A.6) of the Appendix in $[\mathrm{CFM}])$. Hence the natural map $p^{*} p_{*}\left(\mathcal{O}_{S^{\prime}}(2 E)\right)$ $\rightarrow \mathcal{O}_{S^{\prime}}(2 E)$ is surjective and defines a morphism $\pi: S^{\prime} \rightarrow \mathbf{F}_{e}$ over $\mathbf{P}^{1}$ such that $\pi^{*} \mathcal{O}_{\mathbf{F}_{e}}\left(C_{0}\right) \simeq \pi^{*} \mathcal{O}_{\mathbf{F}_{e}}(1) \simeq \mathcal{O}_{S^{\prime}}(2 E)$. The morphism $\pi$ is generically finite of degree 2. Since $\pi^{*}\left(C_{0}\right)=2 E$, the curve $C_{0}$ is a component of the branch locus of $\pi$ and $e=-C_{0}^{2}=-2 E^{2}=2$.

Now

$$
\sigma^{*}\left(K_{S}\right) \equiv 3 \sigma^{*}(C)=3\left(\pi^{*}(F)+E\right) \equiv \pi^{*}\left(C_{0}+3 F\right)+E
$$

and, by the ramification formula, the branch curve $B$ of $\pi$ sits in the linear system $\left|14 F+8 C_{0}\right|$. As we saw, $B=C_{0}+B^{\prime}$, with $B^{\prime}$ a curve, and we compute $B^{\prime} \cdot C_{0}=0$. This yields $B^{\prime} \cap C_{0}=\emptyset$. If $B^{\prime}$ has only non-essential singularities, then $p_{g}=h^{0}\left(\mathbf{F}_{2}, \mathcal{O}_{\mathbf{F}_{2}}\left(C_{0}+3 F\right)\right)=6$ and $K_{S}^{2}=\left(\pi^{*}\left(C_{0}+3 F\right)+E\right)^{2}=9$; therefore $q=0$ by $(0.4, \mathrm{i})$. Since we are assuming $K^{2}=9$, there is no other possibility than the one above. $\diamond$

We want to point out that the surface with $p_{g}=6, K^{2}=9$, presenting the nonstandard case, which we met in the statement of proposition (1.1), already appears in $[\mathrm{D}]$ as well as in $[\mathrm{B}]$, pg. 193. Notice also that, with the above notation, one has $h^{0}\left(S, \mathcal{O}_{S}(2 D)\right)=4$ and the map $\phi_{2 D}$ is a morphism of degree two of $S$ to a quadric cone $Q$ in $\mathbf{P}^{3}$ (see $[\mathrm{CFM}]$, surface of type $\left(H_{1}\right), \S 2$ ).

\section{The CASES $q=1,2$}

We recall that, in view of the quoted results of $[\mathrm{CFM}]$, all non-standard cases with $p_{g} \geq 4$ have $q=0$. Therefore in this section we may limit ourselves to the case $p_{g}=3$ and we will prove the following:

(2.1) Proposition. Let $S$ be a minimal surface of general type with $1 \leq q \leq 2$ and $p_{g}=3$ such that the bicanonical map is not birational. Then $S$ presents the standard case.

Before proving the proposition, we need some preliminaries. Let $S$ be a minimal irregular surface of general type with $p_{g}=3$, such that the bicanonical map is not birational and $S$ presents the non-standard case. By lemma (0.7), if $q \geq 2$ we can exclude the existence of irrational pencils $f: S \rightarrow B$ of genus $b \geq 2$. Hence the image of the Albanese map $a: S \rightarrow A l b(S)$ is a surface. If $\eta \in P i c^{0}(S)$ is a general point, one has $h^{1}(S, \eta)=0$ and $\operatorname{dim}|K+\eta|=\chi\left(\mathcal{O}_{S}\right)-1=3-q$. This is a consequence of (0.5) if $q \geq 2$, and it is obvious for $q=1$. 
If $\eta \in \operatorname{Pic}^{0}(S)$, we will denote by $C_{\eta}$ a curve in $|K+\eta|$. Let us write $|K+\eta|=$ $F_{\eta}+\left|M_{\eta}\right|$, where $F_{\eta}$ is the fixed part of $|K+\eta|$ and $\left|M_{\eta}\right|$ is the movable part. We denote $F_{\eta}$ and $M_{\eta}$ by $F$ and $M$ if no confusion arises.

We will need the following:

(2.2) Lemma. Fix $\eta \in \operatorname{Pic}^{0}(S)$ and let $x, y$ be points on $S$ such that $\phi(x)=\phi(y)$, with $y$ not lying in the base locus of $|K-\eta|$. In particular one may assume that $x, y$ are general points such that $\phi(x)=\phi(y)$. Then $x$ belongs to a curve $C_{\eta}$ in $|K+\eta|$ if and only if $y \in C_{\eta}$.

Proof. Assume that $x \in C_{\eta}$. Then $y \in C_{\eta}+C_{-\eta} \in|2 K|$ for any curve $C_{-\eta} \in|K-\eta|$. Since $y$ does not lie in the base locus of $|K-\eta|$, we have that $y \notin C_{-\eta}$ for $C_{-\eta}$ general in $|K-\eta|$. Hence $y$ sits on $C_{\eta}$. $\diamond$

The above claim certainly applies also to the case $q=3$, but it could have empty meaning. In fact for $\eta \in P i c^{0}(S)$ general, there could be no pair of points $x, y$ on $S$ such that $\phi(x)=\phi(y)$ and such that $x$ lies on the unique curve in $|K+\eta|$ while $y$ does not lie on the unique curve in $|K-\eta|$.

Now we can start the

Proof of proposition (2.1). We argue by contradiction and assume that $S$ presents the non-standard case. We will discuss the two cases $q=1$ and $q=2$ separately. Notice that $K^{2} \geq 6$ by $(0.4)$.

Case $q=2$. We assume first that, for $\eta \in P i c^{0}(S)$ general, the general curve in $\left|M_{\eta}\right|$ is irreducible of arithmetic genus $g$. We notice that $g \geq 3$. This follows by $(0.2)$ or by the following easy argument: we have

$$
2 g-2=(K+M) \cdot M=(F+2 M) \cdot M \geq 2+2 M^{2} ;
$$

hence $g=2$ would imply $M^{2}=0$, and $|M|$ would be a pencil of curves of genus 2, a contradiction.

Let us fix a general point $\eta \in P i c^{0}(S)$ and consider the map $\mu_{\eta}$ which sends the general point $\eta^{\prime} \in \operatorname{Pic}^{0}(S)$ to $\mathcal{O}_{S}\left(M_{\eta^{\prime}}-M_{\eta}\right) \in \operatorname{Pic}^{0}(S)$. Since it depends on $\eta$ only up to a translation, the dimension of its image is independent of $\eta$. Abusing notation, we will simply denote it by $\mu$.

Let us assume first that $\operatorname{dim} \operatorname{Im}(\mu) \leq 1$. This is equivalent to saying that $F_{\eta}$ has some component $G_{\eta}$ which varies with $\eta$ : we denote it by $G$, hoping that there is no danger of confusion. We make our first claim:

Claim 1. If $G$ is as above, then $K \cdot G \leq 3$. In fact if $K \cdot G \geq 4$, then $F \cdot M+M^{2}=$ $K \cdot M=K^{2}-K \cdot F \leq K^{2}-K \cdot G \leq 4$, because by proposition (1.1) we may assume $K^{2} \leq 8$. Since $F \cdot M$ is positive and even, the only possibilities are $F \cdot M=2$, $1 \leq M^{2} \leq 2$, and $F \cdot M=4, M^{2}=0$. In either case we have $\operatorname{dim} \operatorname{Im}(\mu)=0$. Otherwise $M$ would describe a family $\mathcal{M}$ of dimension $r \geq 2$ of curves which is not contained in a linear system. By proposition (0.16) we would have $M^{2}=2$, and by theorem (0.20), since $q \leq 2$, the surface $S$ would not be of general type, a contradiction. Since $\operatorname{dim} \operatorname{Im}(\mu)=0$, there is some curve $F^{\prime}$ contained in $F$, varying in a 2-dimensional family of curves on $S$ not contained in a linear system, and such that all components of $F^{\prime}$ move with it.

If $F \cdot M=4$, one has $K^{2}=8$ and $K \cdot F=K \cdot G=4$. Thus any component $G^{\prime}$ of $F-G$ is such that $K \cdot G^{\prime}=0$; thus $G^{\prime}$ is not movable. Hence $G$ is the only movable component of $F$, and therefore $G=F^{\prime}$. Since the irreducible curve $G$ moves in a 2-dimensional family of curves on $S$ not contained in a linear system, one has 
$G^{2} \geq 3$ (see again proposition (0.16) and theorem (0.20)). On the other hand, the index theorem and $\left(0.4\right.$, i) say that $16=(K \cdot G)^{2} \geq K^{2} \cdot G^{2} \geq 24$, a contradiction.

If $F \cdot M=2$, since $M^{2} \geq 1$ one has $K \cdot M \geq 3$ and therefore $K \cdot F \leq 5$. If $K \cdot G \geq 4$, then any component $G^{\prime}$ of $F-G$ is such that $K \cdot G^{\prime} \leq 1$ and therefore $G^{2} \leq-1$. Thus $G$ is again the only movable component of $F$. Since both $M$ and $G$ move, one has $M \cdot G \geq 0$; but $M \cdot G=0$ is impossible because $M$ moves in a linear pencil and $G$ moves in a non-linear system of dimension 2. Also $M \cdot G=1$ is impossible, because otherwise the curves $G$ would be rational. Since $M \cdot G \leq M \cdot F=2$, one has $M \cdot G=2$.

Consider the rational map

$$
\rho: \eta \in P i c^{0}(S) \rightarrow \mathcal{O}_{M}\left(G_{\eta}\right) \in \operatorname{Pic}^{2}(M) .
$$

Its image, by the above discussion, lands in the image of $M^{(2)}$ in $\operatorname{Pic}^{2}(M)$. By $(0.10, \mathrm{~b})$ the map $\rho$ must be dominant, but this is a contradiction since $M^{(2)}$ is a surface of general type. This ends the proof of claim 1.

Let $G$ be a movable component of $F$. The index theorem and claim 1 yield $9 \geq(K \cdot G)^{2} \geq K^{2} \cdot G^{2} \geq 6 \cdot G^{2}$, namely $G^{2} \leq 1$. The case $G^{2}=1$ is impossible: in fact by proposition (0.18) the surface $S$ would be isomorphic to the symmetric product of a curve of genus $g \geq 3$, which never has invariants $p_{g}=3, q=2$ and $K^{2} \leq 8$. So we have $G^{2}=0$. Since $K \cdot G \leq 3$, one has indeed $K \cdot G=2$ and $S$ would present the standard case, a contradiction which concludes the discussion of the case $\operatorname{dim} \operatorname{Im}(\mu) \leq 1$.

Now we discuss the case $\operatorname{dim} \operatorname{Im}(\mu)=2$. First of all we make:

Claim 2. If $\eta \in \operatorname{Pic}^{0}(S)$ is general, then $M_{\eta}^{2}>0$. Since $M_{\eta}$ is irreducible and moves, the only alternative would be $M_{\eta}^{2}=0$. But this is incompatible with $\operatorname{dim} \operatorname{Im}(\mu)=2$ and $\operatorname{dim}\left|M_{\eta}\right|=1$ (see lemma $(0.14, \mathrm{i})$ ).

By lemma (2.2), the morphism $\phi$ is not birational on a general curve $M_{\eta}$ and the degree of the morphism $\phi: M_{\eta} \rightarrow \Gamma_{\eta}=\phi\left(M_{\eta}\right)$ coincides with the degree $\sigma$ of the bicanonical map. By $(0.5, \mathrm{i})$ one has $h^{1}\left(S, \mathcal{O}_{S}(K-\eta)\right)=0$ if $\eta \in \operatorname{Pic}^{0}(S)$ is general. Then the linear system $\left|K+M_{\eta}-\eta\right|$ cuts out on $M_{\eta}$ a complete linear series $\xi_{\eta}$ of degree $2 g-2$. By claim 2 and $(0.10, \mathrm{~b})$ the linear series $\xi_{\eta}$ is not special; hence it has dimension $g-2$. Since $\left|K+M_{\eta}-\eta\right|$ is contained in $|2 K|$, there is a complete linear series $\gamma_{\eta}$ on $\Gamma_{\eta}$ such that its pull-back via $\phi$ is the movable part of $\xi_{\eta}$. In conclusion we have

$$
\frac{2 g-2}{\sigma} \geq \operatorname{deg}\left(\gamma_{\eta}\right) \geq \operatorname{dim}\left(\gamma_{\eta}\right)=\operatorname{dim}\left(\xi_{\eta}\right)=g-2,
$$

and hence

$$
\sigma \leq 2+\frac{2}{g-2}
$$

Let us first discuss the case $g \geq 5$. Then $\sigma=2$ and the linear series $\gamma_{\eta}$ on $\Gamma_{\eta}$ has dimension $g-2$ and degree at most $g-1$; hence $\Gamma_{\eta}$ has arithmetic genus at most 1. Indeed $\Gamma_{\eta}$ is smooth of genus 1 by $(0.3)$.

Then we consider the linear series $\delta_{\eta, \eta^{\prime}}$ cut out on a general $M_{\eta}$ by the linear system $\left|M_{\eta^{\prime}}\right|$ for $\eta^{\prime} \in P i c^{0}(S)$ general. The generic vanishing $(0.5, \mathrm{i})$ implies again that $\delta_{\eta, \eta^{\prime}}$ is complete. By lemma (2.2), the movable part of $\delta_{\eta, \eta^{\prime}}$ also comes as a pull-back of a complete linear series $\gamma_{\eta, \eta^{\prime}}$ of $\Gamma_{\eta}$. Since $\delta_{\eta, \eta^{\prime}}$ has dimension 1, then

$$
\delta_{\eta, \eta^{\prime}}=\phi^{*}\left(\gamma_{\eta, \eta^{\prime}}\right)+\varphi_{\eta, \eta^{\prime}} \text {, }
$$


where $\gamma_{\eta, \eta^{\prime}}$ is a complete linear series of dimension 1 on $\Gamma_{\eta}$ and $\varphi_{\eta, \eta^{\prime}}$ is the fixed part.

Claim 3. The divisor $\varphi_{\eta, \eta^{\prime}}$, for $\eta^{\prime}$ general, does not depend on $\eta^{\prime} \in \operatorname{Pic}^{0}(S)$. In fact the support of $\varphi_{\eta, \eta^{\prime}}$ is the intersection of $M_{\eta}$ with the base locus of $\left|M_{\eta^{\prime}}\right|$. Under our assumption, i.e. that the general curve $M_{\eta}$ is irreducible, this base locus is finite. Hence the support of $\varphi_{\eta, \eta^{\prime}}$ consists of the points of $S$ which belong to every sufficiently general curve $M_{\eta}$. This clearly implies our assertion.

We can now set $\varphi_{\eta, \eta^{\prime}}=\varphi_{\eta}$. Let us look at the restriction map

$$
\mu^{\prime}: \eta^{\prime} \in \operatorname{Pic}^{0}(S) \rightarrow M_{\eta^{\prime} \mid M_{\eta}} \in \operatorname{Pic}^{0}\left(M_{\eta}\right) \subset \operatorname{Pic}\left(M_{\eta}\right) .
$$

By the above discussion, this coincides, up to a translation, with the map

$$
\rho^{\prime}: \eta^{\prime} \in \operatorname{Pic} c^{0}(S) \rightarrow \phi^{*}\left(\gamma_{\eta, \eta^{\prime}}\right) \in \operatorname{Pic}\left(M_{\eta}\right) .
$$

This yields a contradiction. In fact, the image of the map $\rho^{\prime}$ is of dimension $d \leq 1$ since $\Gamma_{\eta}$ is elliptic, whereas the map $\mu^{\prime}$ has image of dimension 2 by $(0.10, b)$ and by the assumption $\operatorname{dim}(i m \mu)=2$.

Now we discuss the cases $g=3$, 4 . If $g=3$, then (*) yields $2 \leq \sigma \leq 4$. If $\sigma=2$, we conclude as above. If $\sigma=3$, then the linear series $\xi_{\eta}$ is a $g_{4}^{1}$ with a base point $p$. Hence $\gamma_{\eta}$ is a $g_{1}^{1}$ on $\Gamma_{\eta}$, which is therefore rational. This yields that the bicanonical image, possessing the 2-dimensional family of rational curves $\Gamma_{\eta}$, is rational. Hence the curves $M_{\eta}$ would be linearly equivalent, a contradiction. If $\sigma=4$, again we find that $\gamma_{\eta}$ is a $g_{1}^{1}$ on $\Gamma_{\eta}$, and we reach the same contradiction. If $g=4$, then $\xi_{\eta}$ is a $g_{6}^{2}$. If $\xi_{\eta}$ has a base point, then it has two base points because it is composite, and then $M_{\eta}$ is hyperelliptic, contradicting (0.3). If $\xi_{\eta}$ has no base points, then we have $2 \leq \sigma \leq 3$. In any case $\Gamma_{\eta}$ has arithmetic genus at most 1 . The conclusion is then as above.

Finally we have to discuss the case in which the general curve $M_{\eta}$ is reducible. Since $\left|M_{\eta}\right|$ is a linear pencil, it has to be composed with an irrational pencil $f$ : $S \rightarrow B$, with $B$, as we know, of genus 1 . Moreover $M_{\eta} \sim 2 G$, where $G$ is a fibre of $f$. In fact $\mathcal{O}_{S}\left(M_{\eta}\right)$ is the pull-back, via $f$, of a line bundle $\mu_{\eta}$ of degree 2 on $B$. We have $8 \geq K^{2}=K \cdot F_{\eta}+2 K \cdot G \geq 8$, since $K \cdot G \geq 4$ because $S$ presents the non-standard case. The above inequality proves that $K \cdot G=4$ and $K \cdot F_{\eta}=0$, which yields that $F_{\eta}$ does not depend on $\eta$ because it is a union of fundamental cycles. This implies that the map

$$
\eta \in P i c^{0}(S) \rightarrow \mu_{\eta} \in \operatorname{Pic}^{2}(B)
$$

should be generically injective, a contradiction.

Case $q=1$. Since $q=1$, we have $\chi\left(\mathcal{O}_{S}\right)=3$, hence $K^{2} \leq 8<3 \chi\left(\mathcal{O}_{S}\right)$. Since we are in the non-standard case, by applying $(0.4$, ii) we see that the Albanese pencil $a: S \rightarrow \operatorname{Alb}(S)$ is a pencil of hyperelliptic curves of genus 3 and $K^{2}=8$. Since $h^{0}\left(S, \mathcal{O}_{S}(2 K)\right)=11$ and $(2 K)^{2}=32$ and $|2 K|$ is base point free, the degree of the bicanonical map is 2 . Therefore there is an involution $\iota$ on $S$ such that a general pair $(x, y)$ of points of $S$ exchanged by $\iota$ are such that $\phi(x)=\phi(y)$. We refer to $\iota$ as to the bicanonical involution of $S$.

Claim 4. For $\eta$ any point in $\operatorname{Pic}^{0}(S)$ and for any pair $\left(G, G^{\prime}\right)$ of fibres of a, one has $h^{0}\left(S, \mathcal{O}_{S}\left(K+\eta-G-G^{\prime}\right)\right)=0$. Otherwise we have $K \sim 2 G+H$, where $H$ is an effective divisor. Then $8=K^{2}=2 K \cdot G+K \cdot H=8+K \cdot H$, whence $K \cdot H=0$, and the components of $H$ are rational. Therefore $H \cdot G=0$, contradicting the connectedness of the canonical divisors. 
Claim 5. Let $\eta \in \operatorname{Pic}^{0}(S)$ be a general point and let $G$ be a general fibre of the Albanese map a. Then $h^{0}\left(S, \mathcal{O}_{S}(K+\eta-G)\right)>0$, i.e. $K+\eta \equiv G+A_{\eta, G}$, with $A_{\eta, G}$ a curve on $S$. Assume, on the contrary, that $h^{0}\left(S, \mathcal{O}_{S}(K+\eta-G)\right)=0$. Then the restriction map

$$
\rho_{\eta, G}: H^{0}\left(S, \mathcal{O}_{S}(K+\eta)\right) \rightarrow H^{0}\left(G, \omega_{G}\right)
$$

is an isomorphism, since it is injective, and $h^{0}\left(S, \mathcal{O}_{S}(K+\eta)\right) \geq 3$.

Now we make the following remark:

Remark 1. For $\eta \in P i c^{0}(S)$, the fixed divisor $F_{\eta}$ of $|K+\eta|$ is contained in a union of fibres of a but does not contain any fibre of a. Hence it does not depend on $\eta$, for $\eta \in P i c^{0}(S)$ a general point. Since $\rho_{\eta, G}$ is an isomorphism, we have $F_{\eta} \cdot G=0$, and therefore $F_{\eta}$ is contained in a union of fibres of $a$. Assume that $F_{\eta}=G_{\eta}+F_{\eta}^{\prime}$, with $G_{\eta}$ a fibre, and $F_{\eta}^{\prime}$ not containing any fibre by claim 4 . Then

$$
\begin{gathered}
8=K^{2}=K \cdot G_{\eta}+K \cdot F_{\eta}^{\prime}+K \cdot M_{\eta} \\
=4+K \cdot F_{\eta}^{\prime}+G_{\eta} \cdot M_{\eta}+M_{\eta} \cdot F_{\eta}^{\prime}+M_{\eta}^{2}=8+K \cdot F_{\eta}^{\prime}+M_{\eta} \cdot F_{\eta}^{\prime}+M_{\eta}^{2}
\end{gathered}
$$

and hence $K \cdot F_{\eta}^{\prime}=M_{\eta} \cdot F_{\eta}^{\prime}=M_{\eta}^{2}=0$. Therefore $\left|M_{\eta}\right|$ is composed of a pencil, whose general curve we denote by $L$, i.e. $M_{\eta} \sim i L, i \geq 2$. Since $M_{\eta} \cdot G=4$, and since the only irrational pencil on $S$ is the Albanese pencil, we have that $|L|$ is a rational pencil, hence $i=2$. Since $K \cdot M_{\eta}=4$, we would have $K \cdot L=2$, contradicting the fact that we are in a non-standard case. This proves the remark.

As a consequence we have

Remark 2. For $\eta \in \operatorname{Pic}^{0}(S)$ general the linear system $|K+\eta|$ is not composed of a pencil. Suppose to the contrary that $\left|M_{\eta}\right|$ is composed of a pencil, so that $\left|M_{\eta}\right|=$ $\left|i L_{\eta}\right|$, with $i \geq 2$. Since $M_{\eta} \cdot G=4$, we have $i=2$ and $\left|L_{\eta}\right|$ is a rational pencil. Then $8=K^{2}=K \cdot F_{\eta}+2 K \cdot L_{\eta} \geq 2 K \cdot L_{\eta}$ implies $4 \geq K \cdot L_{\eta}=F_{\eta} \cdot L_{\eta}+2 L_{\eta}^{2} \geq 1+2 L_{\eta}^{2}$. Hence $1 \geq L_{\eta}^{2} \geq 0$. By the remark above, the curve $L_{\eta}$ varies, as $\eta$ varies in $\operatorname{Pic}^{0}(S)$ in a 2-dimensional irreducible system of curves on $S$, not contained in a linear system, whose general curve is irreducible. By lemma (0.14) and proposition (0.16) we find a contradiction.

Now there are two cases to be considered according to whether the bicanonical involution $\iota$ fixes the general fibre $G$ of the Albanese map $a$ or not. First we suppose that $\iota$ does not fix the general fibre $G$ of $a$. Let $G^{\prime}=\iota(G) \neq G$. If $D$ is a general canonical divisor on $G$, let $D^{\prime}=\iota(D)$ be the corresponding divisor on $G^{\prime}$. By remark 2 above, the general curve $M_{\eta}$ is irreducible. Then for the general $\eta \in P i c^{0}(S)$ there is one single curve in $\left|M_{\eta}\right|$ containing $D$ and $D^{\prime}$, and the general such curve is irreducible. Let $\mathcal{M}$ be the family described by these curves, which is parametrized by $\operatorname{Pic}^{0}(S)$. Since $M_{\eta}^{2} \leq K^{2}=8$ and since the degree of $D+D^{\prime}$ is 8 , two general curves of $\mathcal{M}$ only meet at the base points $D$ and $D^{\prime}$. Hence $\mathcal{M}$ is a pencil with base points on $S$, i.e. it is a rational pencil, contradicting remark 1 .

Next we consider the case in which $\iota$ fixes the general fibre $G$ of the Albanese map $a$.

The map $\phi_{K+\eta}: S \rightarrow \mathbf{P}^{2}$, which is surjective by remark 2 , restricts on $G$ to the canonical map. Hence the curves of the Albanese fibration are mapped by $\phi_{K+\eta}$ to conics in the plane. We claim that $\operatorname{deg}\left(\phi_{K+\eta}\right)>2$. Otherwise let $\mathcal{C}$ be the family of conics in the plane which are images of the fibres of $a$ via $\phi_{K+\eta}$, let $p$ be a general point in $\mathbf{P}^{2}$ and let $x, y$ be the two points of $S$ such that $\phi_{K+\eta}(x)=\phi_{K+\eta}(y)=p$. 
Then, by our assumption on $\iota$, we have that $x, y$ belong to a unique fibre $G$ of $a$, and the conic $\phi_{K+\eta}(G)$ would be the unique curve of $\mathcal{C}$ passing through $p$. Hence $\mathcal{C}$ would be a linear pencil, which in turn would imply that the fibres of $a$ are linearly equivalent, a contradiction.

But if $\operatorname{deg}\left(\phi_{K+\eta}\right)>2$, with the same argument as above we also come to a contradiction by finding a system $\mathcal{M}$ of curves parametrized by $\mathrm{Pic}^{0}(S)$, with base points and no variable intersections. Claim 5 is thus proved.

Finally we prove the following claim, which contradicts claim 4:

Claim 6. The curve $A:=A_{\eta, G}$ contains some fibre of the Albanese map. Since $A \cdot G=4$, we have $A^{2}=0$. Since $A$ moves on $S$ we can write $A=B+C$, where $B$ is the fixed part and $C$ the movable part. Let $C^{2}>0$. Since $K \cdot C \leq 4$, by the index theorem the only possible cases are either $C^{2}=1$ and $K \cdot C=3$ or $C^{2}=2$ and $K \cdot C=4$. The former case can be excluded by using, as we did already before, proposition (0.18). In the latter case we have $K \cdot B=0$; hence $B \cdot G=0$ and $C \cdot G=A \cdot G=4$. Then $4=K \cdot C=C^{2}+C \cdot B+C \cdot G \geq 6$, a contradiction. Therefore we have $C^{2}=0$. The assertion follows from the fact that $A$ moves in a non-linear system and the Albanese pencil is the only irrational pencil on $S$. $\diamond$

\section{THE CASE $q=3$}

Again we recall that, by $[\mathrm{CFM}]$, all non-standard cases with $p_{g} \geq 4$ have $q=0$. Hence we may assume $p_{g}=3$. In this section we are going to classify the smooth minimal surfaces $S$ with $p_{g}=q=3$ and with the bicanonical map $\phi$ not birational. First we will deal with the non-standard case. In view of proposition (1.1) and $(0.4, \mathrm{i})$, we may assume that $8 \geq K^{2} \geq 6$. First we will exclude the cases $K^{2}=7,8$, and then we will prove in proposition (3.22) that if $S$ is a surface with $p_{g}=q=3$ and $K^{2}=6$, then $S$ is the symmetric product of a curve of genus 3 . This proves the first part of theorem B of the introduction. Finally we will classify the standard cases, concluding the proof of theorem B.

Notice that $h^{0}\left(S, \mathcal{O}_{S}(2 K)\right)=K^{2}+1$; hence the bicanonical image $\Sigma$ of $S$ is a surface of degree $\operatorname{deg}(\Sigma)=\frac{4 K^{2}}{\sigma}$ in $\mathbf{P}^{K^{2}}$. This shows that $\sigma \leq 4$ and that $\sigma$ is even if $K^{2}>6$.

First of all we prove

(3.1) Proposition. Let $S$ be a surface of general type with $p_{g}=q=3$. Then:

(i) If there is an irrational pencil $f: S \rightarrow B$ with $B$ a smooth curve of genus $b$ and fibres of genus $g$, then either $b=1$ and $g \geq 3$, or $b=g=2, K^{2}=8$, the map $f$ is smooth and the curves of the pencil have constant modulus.

(ii) The image of the Albanese map $a: S \rightarrow S^{\prime} \subseteq A l b(S)$ is a surface.

Let $\Delta$ be the subscheme of $S$ where a drops rank, and suppose there is no irrational pencil $f: S \rightarrow B$ with $B$ a smooth curve of genus $b=2$. Then:

(iii) The natural map $w: \wedge^{2} H^{0}\left(S, \Omega_{S}^{1}\right) \rightarrow H^{0}\left(S, \Omega_{S}^{2}\right)$ is an isomorphism, and $\Delta$ is the base point scheme of $|K|$ and is also the scheme defined by the condition that $\Omega_{S}^{1}$ is not generated by the global sections there. In particular any curve of $S$ contracted by a, e.g. any rational curve, is contained in the base locus of $|K|$. Moreover, if the differential of $a$ is zero at $p \in \Delta$, then $p$ is a multiple base point for $|K|$;

(iv) If, in addition, $|K|$ has no fixed component, then $\Delta$ is finite of length $\delta=$ $2\left(K^{2}-6\right)$. 
(v) If, in addition, $\phi:=\phi_{2 K}$ is not birational, then either $K^{2}=6$ and $|K|$ is base point free, or $K^{2}=8$, and this is the only case in which $|K|$ may have a base point of multiplicity 2. If $K^{2}=8$ and $|K|$ has a base point of multiplicity 2, then there is a curve $K_{0} \in|K|$ having at $p$ a point of multiplicity at least 3.

Proof. Let us prove (i). If $b=1$ the assertion follows by the last part of (0.6). Suppose $b \geq 3$. By $(0.6)$ we would get $K^{2} \geq 16$, contradicting the Miyaoka-Yau inequality, which in the present case reads out as $K^{2} \leq 9$. If $b=2$, arguing as above and using (0.6) again, we have that the only possibility is as stated. We observe that (ii) follows by (i) since, if the image of $a$ were a curve, its genus would be 3 .

In order to prove (iii) we remark that if $w$ is not an isomorphism, then there is a non-zero indecomposable tensor $\omega_{1} \wedge \omega_{2} \in \wedge^{2} H^{0}\left(S, \Omega_{S}^{1}\right)$ in the kernel of $w$. According to Castelnuovo-De Franchis' theorem ([BPV], pg. 123), there is then an irrational pencil $f: S \rightarrow B$ with $B$ a smooth curve of genus $b \geq 2$. By (i) and by the assumption we have a contradiction.

Let $p$ be a point of $S$ and let $(x, y)$ be coordinates with origin at $p$. Let $\left(\omega_{1}, \omega_{2}, \omega_{3}\right)$ be a basis of $H^{0}\left(S, \Omega_{S}^{1}\right)$, and write $\omega_{i}=f_{i}(x, y) d x+g_{i}(x, y) d y, i=1,2,3$, around $p$. The equations of $\Delta$ around $p$ are

$$
r k\left(\begin{array}{lll}
f_{1} & f_{2} & f_{3} \\
g_{1} & g_{2} & g_{3}
\end{array}\right) \leq 1
$$

It is clear that these same equations define the scheme where $\Omega_{S}^{1}$ is not generated by the global sections. In view of the isomorphism $w: \wedge^{2} H^{0}\left(S, \Omega_{S}^{1}\right) \rightarrow H^{0}\left(S, \Omega_{S}^{2}\right)$, a basis of $H^{0}\left(S, \Omega_{S}^{2}\right)$ is given by $\left(w\left(\omega_{i} \wedge \omega_{j}\right)\right)_{1 \leq i<j \leq 3}$. This shows that the base locus of $|K|$ is given around $p$ by the same equations as $\Delta$ and that if $a$ has zero differential at $p$, then $p$ is a multiple base point for $|K|$. This proves (iii).

As for (iv), we assume $|K|$ has no fixed component. By (0.1), the general canonical curve is irreducible and reduced. The choice of the basis $\left(\omega_{1}, \omega_{2}, \omega_{3}\right)$ of $H^{0}\left(S, \Omega_{S}^{1}\right)$ determines a map $\mathcal{O}_{S}^{3} \rightarrow \Omega_{S}^{1}$, whose cokernel we denote by $\mathcal{T}$. By theorem 1.22 of $[\mathrm{Ca}]$ one has length $(\mathcal{T})=K^{2}-c_{2}(S)=2 K^{2}-12$. We now prove that $\delta:=$ length $(\Delta)=$ length $(\mathcal{T})$. Working locally, and using the Hilbert-Burch theorem, we see that there is an exact sequence

$$
0 \rightarrow T_{S} \rightarrow \mathcal{O}_{S}^{3} \rightarrow \mathcal{I}_{\Delta}(K) \rightarrow 0,
$$

where $T_{S}$ is the tangent sheaf of $S$. Dualizing the above sequence, we get

$$
0 \rightarrow \mathcal{O}_{S}(-K) \rightarrow \mathcal{O}_{S}^{3} \rightarrow \Omega_{S}^{1} \rightarrow \mathcal{T} \rightarrow 0 .
$$

Therefore we have $\mathcal{T} \simeq \operatorname{Ext}^{1}\left(\mathcal{I}_{\Delta}(K), \mathcal{O}_{S}\right) \simeq \operatorname{Ext}^{2}\left(\mathcal{O}_{\Delta}, \mathcal{O}_{S}(-K)\right)$. By local duality $\operatorname{Ext}^{2}\left(\mathcal{O}_{\Delta}, \mathcal{O}_{S}\right)^{*} \simeq \operatorname{Hom}\left(\omega_{S}, \mathcal{O}_{\Delta}\right) \simeq \mathcal{O}_{\Delta}$, which proves our assertion.

The first part of (v) follows by (iv) and the fact that if $K^{2}=7$, then $|K|$ yields a map of degree five to $\mathbf{P}^{2}$. Since $\sigma \leq 4$, we find a contradiction.

Let us now prove the second assertion of $(\mathrm{v})$. Let $\mathbf{m}_{p}$ be the maximal ideal of the local ring of $S$ at $p$. We have a natural map

$$
r: H^{0}\left(S, \mathcal{O}_{S}\left(K_{S}\right)\right) \rightarrow \frac{\mathbf{m}_{p}^{2}}{\mathbf{m}_{p}^{3}}
$$

Were $r$ surjective, then the length of the base point scheme $\Delta$ of $|K|$ at $p$ would be 3, contradicting (3.1). Then $r$ is not surjective, and also not injective because $h^{0}\left(S, \mathcal{O}_{S}\left(K_{S}\right)\right)=\operatorname{dim} \frac{\mathbf{m}_{p}^{2}}{\mathbf{m}_{p}^{3}}=3$. This completes the proof of part $(\mathrm{v}) . \diamond$ 
In our discussion we will distinguish two main cases: (i) $|K|$ has a fixed part; (ii) $|K|$ has no fixed part, and, according to (0.1) and (3.1), the general canonical curve is irreducible and reduced and $K^{2}=6,8$. Let us prove the following lemmas:

(3.2) Lemma. Let $S$ be a minimal surface of general type with $p_{g}=q=3$ and no irrational pencils of curves of genus 2. Suppose that $L<K$ is an effective divisor such that $K \cdot L \leq 7$ and $h^{0}\left(S, \mathcal{O}_{S}(L)\right)=2$. If $|L|=\Phi+|V|$, where $\Phi$ is the fixed part of $|L|$ and $|V|$ is the moving part, then the general curve in $|V|$ is irreducible and $K \cdot V+V^{2} \geq 8$. In particular, $K \cdot V \geq 5$. If, in addition, $K^{2}=8$ and $\phi$ is not birational, then $K \cdot V \geq 6$.

Proof. Suppose that the general curve in $|V|$ is reducible. Then $|V|$ is composed with an irrational pencil pencil $f: S \rightarrow B$, and $V \sim m D$, where $D$ is a fibre of $f$ and $m \geq 2$. Since $K \cdot D$ is even and $2 K \cdot D \leq m K \cdot D=K \cdot V \leq 7$, we get $K \cdot D=2$ and the pencil has fibres of genus 2 , a contradiction.

Therefore the general curve in $|V|$ is irreducible. By $(0.2)$ we have $K \cdot V+V^{2} \geq 8$. Since $K \cdot V=V^{2}+V \cdot \Phi$, we have $2 K \cdot V \geq 8+V \cdot \Phi$, and by 2-connectedness of the canonical divisors we get the assertion.

Suppose that $K^{2}=8, \phi$ is not birational and $K \cdot V=5$, so that $V^{2} \geq 3$. Set $\Psi=K-V$. Hence $5=K \cdot V=V^{2}+V \cdot \Psi \geq V^{2}+2 \geq 5$, and therefore $V \cdot \Psi=2$ and $V^{2}=3$. From $8=K^{2}=\Psi^{2}+2 V \cdot \Psi+V^{2}=\Psi^{2}+7$ we deduce that $\Psi^{2}=1$. Also, $\Psi$ is 1 -connected by $(0.11)$. Hence $h^{1}\left(S, \mathcal{O}_{S}\left(K_{S}+\Psi\right)\right)=0$. Let $V$ be general in $|V|$. Since $V^{2}=3$, the linear system $|V|$, which has no fixed components, has also no multiple base points; hence $V$ is smooth. From the exact sequence

$$
0 \rightarrow \mathcal{O}_{S}(K+\Psi) \rightarrow \mathcal{O}_{S}(2 K) \rightarrow \mathcal{O}_{V}(2 K) \rightarrow 0
$$

it follows that $|2 K|$ cuts out on $V$ a complete, composed, linear series of degree $10=2 p_{a}(V)$. This implies that $V$ is hyperelliptic, contradicting $(0.3) . \diamond$

(3.3) Lemma. Let $S$ be a minimal surface of general type with $p_{g}=q=3$. Suppose that $|K|$ has a fixed part, and write $|K|=F+|M|$, where $F$ is the fixed part of $|K|$ and $|M|$ is the movable part. Then:

(i) the general curve $M \in|M|$ is irreducible, $M^{2} \geq 3$, and $h^{0}\left(S, \mathcal{O}_{S}(K+M)\right)=$ $p_{a}(M) \geq 6$. Moreover, if $K^{2}=6$ then $M^{2} \geq 4$;

(ii) if $K^{2}=8$ then $K$ is ample, and if $K^{2}=7$ there is at most one fundamental cycle on $S$, which can be of the type $\left(A_{i}\right), i=1,2$;

(iii) if $M^{2} \geq 4$ one has the following numerical possibilities:

$\begin{array}{cccccccc} & K^{2} & K \cdot F & K \cdot M & F \cdot M & F^{2} & M^{2} & p_{a}(M) \\ \left(A_{1}\right) & 8 & 2 & 6 & 2 & 0 & 4 & 6 \\ \left(A_{2}\right) & 7 & 1 & 6 & 2 & -1 & 4 & 6 \\ \left(B_{1}\right) & 7 & 0 & 7 & 2 & -2 & 5 & 7 \\ \left(B_{2}\right) & 8 & 1 & 7 & 2 & -1 & 5 & 7 \\ (C) & 6 & 0 & 6 & 2 & -2 & 4 & 6\end{array}$

(iv) in the cases $(A)$ and $(C)$ of the table $F$ is the fixed part of $|K+M|$, and in the cases $(B)$ the image of restriction map $H^{0}\left(S, \mathcal{O}_{S}(K+M)\right) \rightarrow H^{0}\left(F, \mathcal{O}_{F}(K+M)\right)$ is at most 1-dimensional;

(v) if $M^{2}=4, K \cdot M=6$, and if there is no irrational pencil of curves of genus 2 on $S$, then the linear system $|M|$ is base point free and $F$ coincides with the fixed locus of $|K+M|$; 
(vi) if $K^{2}=7,8$ and the map $\phi$ is not birational, one has $M^{2} \geq 4$. Then either we are in cases $(A)$ of the table above and the linear system $|M|$ is base point free, or we are in cases $(B)$ and then $|M|$ has one simple base point. In both cases the general curve in $|M|$ is non-singular.

Proof. The first assertion of (i) follows by (0.1). By (0.3) we have $M^{2} \geq 3$. Hence $h^{1}\left(S, \mathcal{O}_{S}(K+M)\right)=0$. Then the theorem of Riemann-Roch yields

$$
h^{0}\left(S, \mathcal{O}_{S}(K+M)\right)=p_{a}(M)
$$

and one has

$$
p_{a}(M)=h^{0}\left(S, \mathcal{O}_{S}(K+M)\right) \geq h^{0}\left(S, \mathcal{O}_{S}(2 M)\right) \geq \operatorname{dimSym}^{2} H^{0}\left(S, \mathcal{O}_{S}(M)\right)=6 .
$$

Remark that $F \cdot M$ is an even positive integer because $K \cdot M+M^{2}=M \cdot F+2 M^{2} \equiv$ $0(\bmod 2)$. Moreover $K^{2}=K \cdot F+F \cdot M+M^{2}=K \cdot F+K \cdot M$ and $K$ is nef. If $K^{2}=6$ one has $F \cdot M+M^{2}=K \cdot M \leq 6$, and $M^{2} \geq 3$ yields $M \cdot F=2$. Since $p_{a}(M) \geq 6$ we have $M^{2} \geq 4$, completing the proof of (i).

Assertion (ii) follows from (0.8), and the table in (iii) follows easily by (ii), the hypothesis $M^{2} \geq 4$ and the fact that $M \cdot F$ is a positive even integer.

By (i) we have $h^{0}\left(S, \mathcal{O}_{S}(K+M)\right)=6$ for $(\mathrm{A})$ and $(\mathrm{C})$ and $h^{0}\left(S, \mathcal{O}_{S}(K+M)\right)=7$ for $(\mathrm{B})$. On the other hand,

$$
h^{0}\left(S, \mathcal{O}_{S}(K+M-F)\right)=h^{0}\left(S, \mathcal{O}_{S}(2 M)\right) \geq \operatorname{dimSym}^{2} H^{0}\left(S, \mathcal{O}_{S}(M)\right)=6,
$$

and thus we have (iv).

Let us now prove $(\mathrm{v})$, for which it suffices to prove that $|M|$ is base point free. Suppose this is not the case. Then, by $(0.3),|M|$ has one simple base point $p$. By (i) and (iii) we have $h^{0}\left(S, \mathcal{O}_{S}(K+M)\right)=6$ and by $(3.4)$ we get $h^{0}\left(S, \mathcal{O}_{S}(2 M)\right)=$ $\operatorname{dim} \operatorname{Sym}^{2} H^{0}\left(S, \mathcal{O}_{S}(M)\right)=6$. Therefore $p$ is a base point of $|2 M|$, and thus of $|K+M|$ by (iv). Since $p$ is a base point of $|M|$ and $h^{0}\left(S, \mathcal{O}_{S}(M)\right)=3$, there exists a curve $M^{\prime}$ in $|M|$ with a double point at $p$. Then, applying $(0.12, a)$ to $M^{\prime}$, we obtain a decomposition $M^{\prime}=A+B$ where $A, B$ are curves such that $A \cdot B=1$, $p \in A \cap B, p$ a non-singular point of $A, \mathcal{O}_{A}(p) \simeq \mathcal{O}_{A}(B)$, and either $A \cap B=\{p\}$, in which case also $p$ is a non-singular point of $B$, or $A \subset B$.

By 2-connectedness of the canonical divisors and $M \cdot F=2$ we get $A \cdot F=B \cdot F=$ 1. Hence, since $M \cdot A \geq 1$ and $M \cdot B \geq 1$, we have the following possibilities:

(a) $A^{2}=0, M \cdot A=1, K \cdot A=2, B^{2}=2, M \cdot B=3, K \cdot B=4$;

(b) $A^{2}=1, M \cdot A=2, K \cdot A=3, A \sim B$.

Case (a) cannot occur. In fact since $A$ is 1 -connected by $(0.11), M \cdot A=1$ and $p \in A$ yield that the image of the restriction map $H^{0}\left(S, \mathcal{O}_{S}(M)\right) \rightarrow H^{0}\left(A, \mathcal{O}_{A}(M)\right)$ is 1 -dimensional and thus $h^{0}\left(S, \mathcal{O}_{S}(B)\right)=2$. But $K \cdot B=4$, and so we have a contradiction to $(3.2)$.

As for case (b), let us notice that if $\Gamma$ is an irreducible component of $A$ such that $\Gamma \cdot M \neq 0$, then $1 \leq \Gamma \cdot M=2 \Gamma \cdot A \leq A \cdot M=2$; hence $\Gamma \cdot M=2$ and there is only one such a component $\Gamma$ of $A$. Now remark that by $(3.2)$ we have $h^{0}\left(S, \mathcal{O}_{S}(B)\right)=1$ and therefore $h^{0}\left(A, \mathcal{O}_{A}(M)\right) \geq 2$. Since $p$ is a non-singular point of $A, p$ is a base point of $|M|$ and $p_{a}(A)>0$, the fact that $h^{0}\left(A, \mathcal{O}_{A}(M)\right) \geq 2$ implies that the curve $A$, which is 1-connected by (0.11), is not 2-connected. But then $A=C+D$, where $C, D$ are curves such that $C \cdot D=1$, and say $\Gamma \leq C$. Then $M \cdot C=2, M \cdot D=0$, yielding $C^{2}=0, D^{2}=-1$. Since $K \cdot A=3$ we have $K \cdot C=2, K \cdot D=1$. By (0.9) there is an irrational pencil $f: S \rightarrow B$ such that a multiple $m C$ of $C$ is a 
curve of the pencil. Since there is no irrational pencil of curves of genus 2 on $S$, we have $m \geq 2$, which, in view of the presence on $S$ of the curve $D$, contradicts $(0.21)$. Therefore also case (b) cannot occur, and hence $|M|$ is base point free.

Finally, if $K^{2}=7,8$ and $\phi$ is not birational, we have $\sigma$ even, excluding $M^{2}=3$. In cases $(\mathrm{A})$ of the table in (iii) the system $|M|$ is base point free by (v). In cases (B), since $\sigma$ is even, $|M|$ must have a simple base point. The last assertion follows by the theorem of Bertini. $\diamond$

Now we first exclude the cases (A) and (B) of the table in (iii) of lemma (3.3); then we will exclude also the case $K^{2}=8$ and $|K|$ with no fixed components. Finally we will discuss the case $K^{2}=6$, proving that $S$ is then the symmetric product of a curve of genus 3 .

Case $\phi$ not birational, $K^{2}=7,8$, and $|K|$ having a fixed component.

We now assume $\phi$ not birational, and we first discuss, and exclude, the cases $(A)$ in the table of lemma (3.3, iii). Then $|M|$ has no base points by lemma $(3.3$, v, vi). Furthermore, we have

(3.5) Lemma. In case $\left(A_{1}\right)$ there is a pencil $f: S \rightarrow E$ with elliptic base $E$, and a multiple $m F$ of $F$, with $m \geq 2$, is a curve of the pencil.

Proof. The curve $F$ is 1-connected by $(0.11)$. Since $p_{a}(F)=2$, it follows that $h^{1}\left(S, \mathcal{O}_{S}(-F)\right) \neq 0$, and $(0.9)$ yields the existence of an irrational pencil $f: S \rightarrow E$ such that a multiple $m F$ of $F$ is a curve of the pencil. The rest of the assertion follows by $(0.7)$. $\diamond$

We can now prove

(3.6) Lemma. In case $\left(A_{1}\right)$ the fixed curve $F$ is irreducible, and in case $\left(A_{2}\right)$ it is 2-connected.

Proof. Case $\left(A_{1}\right)$. By lemma (3.3, ii) we have that $K$ is ample and $K \cdot F=2$. So, if $F$ is reducible, one has $F=F_{1}+F_{2}$ with $K \cdot F_{i}=1$, and $F_{i}$ is irreducible, $i=1,2$. The index theorem says that $F_{i}^{2} \leq-1$, hence either $F_{i}^{2}=-1$ or $F_{i}^{2}=-3$. By lemma $(3.5)$ we have $0=F \cdot F_{i}, i=1,2$; then $F_{1}^{2}=F_{2}^{2}=-F_{1} \cdot F_{2}$. Therefore we have the following possibilities:

$$
\begin{array}{cccc} 
& F_{1}^{2} & F_{2}^{2} & F_{1} \cdot F_{2} \\
\text { (i) } & -1 & -1 & 1 \\
\text { (ii) } & -3 & -3 & 3
\end{array}
$$

Case (i) cannot happen. Indeed since $F F_{i}=0, K F_{i}=M F_{i}$, whence $M \cdot F_{1}=$ $M \cdot F_{2}=1$, which is excluded since $|M|$ is base point free (see lemma $(3.3, \mathrm{v}, \mathrm{vi})$ ) and the $F_{i}$ 's are elliptic.

In case (ii) we apply (0.6) and get

$$
4=c_{2}(S) \geq 1+2 m
$$

hence $m=1$, a contradiction.

Case $\left(A_{2}\right)$. Suppose $F$, which is 1-connected by $(0.11)$, is not 2-connected. Then $F=F_{1}+F_{2}$, with $F_{1} \cdot F_{2}=1$ and, say, $K \cdot F_{1}=1, K \cdot F_{2}=0$. Since $-1=F^{2}=F_{1}^{2}+F_{2}^{2}+2$, we must have $F_{1}^{2}=-1, F_{2}^{2}=-2$. By 2-connectedness of the canonical divisors and since $M \cdot F=2$, we have $M \cdot F_{1}=M \cdot F_{2}=1$. Since $F_{1} \cdot\left(K-F_{1}\right)=F_{1} \cdot M+F_{1} \cdot F_{2}=2$, the curve $F_{1}$ is 1 -connected by $(0.11)$. Since $M$ has no base points, this yields $h^{0}\left(F_{1}, \mathcal{O}_{F_{1}}(M)\right) \geq 2$, and therefore, as 
$p_{a}\left(F_{1}\right)=1$, the curve $F_{1}$ is reducible and not 2-connected (see [CFM], proposition (A.5)). Therefore $F_{1}=G_{1}+G_{2}$ with $G_{1} \cdot G_{2}=1$ and, say, $K \cdot G_{1}=1, K \cdot G_{2}=0$, $G_{1}^{2}=-1, G_{2}^{2}=-2$. Since $F_{1} \cdot F_{2}=1$ and $F_{1} \cdot G_{2}=G_{1} \cdot G_{2}+G_{2}^{2}=-1$, we have $F_{2} \neq G_{2}$. By lemma (3.3, ii), we must have that $F_{2}$ and $G_{2}$ are (-2)-curves meeting transversally at one point. Then, since $1=F_{2} \cdot F_{1}=F_{2} \cdot G_{2}+F_{2} \cdot G_{1}=1+F_{2} \cdot G_{1}$, we have $G_{1} \cdot F_{2}=0$. By 2-connectedness of $K$, one has $M \cdot G_{1}=M \cdot F_{2}=1$, $M \cdot G_{2}=0$. Then $G_{1}$, which is 1 -connected by $(0.11)$, is not 2 -connected and contains a curve of arithmetic genus zero which is met at one point by the curves of $|M|$, i.e. it contains $F_{2}$ and we have $G_{1}=F_{2}+H$, with $H$ effective and $H \cdot F_{2}=1$. But then $G_{1} \cdot F_{2}=F_{2}^{2}+F_{2} \cdot H=-2+1=-1$, a contradiction. $\diamond$

(3.7) Corollary. In the cases $(A)$, one has $h^{0}\left(S, \mathcal{O}_{S}(M-F)\right)=1$. Furthermore if $M^{\prime}$ is the unique curve in $|M|$ containing $F$, it decomposes as $M^{\prime}=A+B$ with $A, B$ curves such that $A \cdot B=1, A \cdot F=B \cdot F=1$, and one has the following possibilities for the decomposition:

$\begin{array}{lccccc} & M \cdot A & A^{2} & M \cdot B & B^{2} & \\ \text { (a) } & 0 & -1 & 4 & 3 & \\ (b) & 1 & 0 & 3 & 2 & \\ (c) & 2 & 1 & 2 & 1 & A \sim B\end{array}$

Proof. Since $F$ is 2-connected with $p_{a}(F) \geq 1, M$ is nef and $M \cdot F=2$, one has that the image of the restriction map $H^{0}\left(S, \mathcal{O}_{S}(M)\right) \rightarrow H^{0}\left(F, \mathcal{O}_{F}(M)\right)$ is at most 2-dimensional. But $|M|$ being base-point free forces this image to be 2-dimensional, so we have the first part of the assertion.

Any intersection point of $F$ with the curve $M^{\prime}-F$ is a multiple point of $M^{\prime}$, and by lemma $(3.3$, iv) it is a base point of $|K+M|$. Then the assertion about the splitting $M=A+B$ with $A \cdot B=1$ follows by $(0.12$, a). The fact that $A \cdot F=$ $B \cdot F=1$ follows from 2-connectedness of the canonical divisors and $M \cdot F=2$. Since $4=M^{2}=A^{2}+B^{2}+2 A \cdot B=A^{2}+B^{2}+2$ and $0 \leq M \cdot A=A^{2}+A \cdot B=A^{2}+1$ and similarly for $B$, one easily verifies that the only possibilities are those listed in table (3.8). $\diamond$

(3.9) Lemma. Also in case $\left(A_{2}\right)$ the curve $F$ is irreducible.

Proof. Assume that $F$ is reducible, and let us consider the map

$$
H^{0}\left(S, \mathcal{O}_{S}(K+F)\right) \rightarrow H^{0}\left(F, \omega_{F}\right) \simeq H^{0}\left(F, \mathcal{O}_{F}\right) \simeq \mathbf{C} .
$$

It must be surjective, as otherwise $F$ would be in the fixed part of $|K+F|$, and since $F$ has some singular point, this is against theorem $(0.12, a)$ and the 2 -connectedness of $F$. Therefore $h^{0}\left(S, \mathcal{O}_{S}(K+F)\right)=4$ and $|K+F|=|2 F+M|$ is base point free, since $|M|$ is base point free.

Now we look at the morphism $\phi_{K+F}$, which contracts $F$ to a point $p$. Since $(K+F)^{2}=8$, its degree is either 4 or 2 . If it is 4 , it maps $S$ to a quadric $Q$. We notice that $p$ is a smooth point of $Q$. Otherwise, since on composing $\phi_{K+F}$ with the projection of $Q$ from $p$ to a plane we have the rational map $\phi_{K}=\phi_{M}$, we would have a contradiction to lemma $(3.3$, i). Let $|L|$ be the linear system on $S$ whose general member is the strict transform via $\phi_{K+F}$ of a general line of a ruling of $Q$. Clearly $L \cdot F=0$, because $F$ is contracted to a smooth point of $Q$. Moreover, since $L \cdot(K+F)=4$, one has $K \cdot L=4$. This contradicts lemma (3.2), because $L<M<K$. This proves that the degree of $\phi_{K+F}$ cannot be 4 . 
Suppose that the degree of $\phi_{K+F}$ is 2 . Then the degree of $\phi_{K+F}$ on a curve of $|M|$ is also 2. On the other hand, since we have equality in formula (3.4) and the degree of $\phi_{M}$ is 4 , the degree of $\phi_{2 M}$ is also 4 . Since, by (3.3, iv) one has $|K+M|=|2 M|+F$ and $|K+F|+(M-F)$ is contained in $|K+M|$, we find a contradiction. $\diamond$

We explicitly notice that, since $F$ is irreducible in both cases $(A)$, by proposition (3.1, iii) there are no smooth rational curves on $S$.

Now we are ready to prove

(3.10) Proposition. The cases $(A)$ cannot occur.

Proof. Case $\left(A_{1}\right)$. Suppose that (a) of table (3.8) happens. Since $K \cdot A=F \cdot A=1$ and, as noted above, there is no smooth rational curve on $S$, the curve $A$ will be irreducible with $p_{a}(A)=1$, and the pencil $f: S \rightarrow E$, which exists by lemma (3.5), induces a dominant morphism $f: A \rightarrow E$, contradicting (0.21).

Also (b) cannot occur. In fact since $M \cdot A=1$, and since $|M|$ is base point free, $A$ should have some smooth rational component, whereas there is no smooth rational curve on $S$.

In case (c) we have $K \cdot A=K \cdot B=3$. Now either $F \leq A$ or $F \leq B$, say $F \leq B$. Since $F \cdot(B-F)=1,(B-F)^{2}=-1, K \cdot(B-F)=1$, again we have an irreducible curve of arithmetic genus 1 as in case (a); hence we get a contradiction.

Case $\left(A_{2}\right)$. If (a) happens, as in the case $\left(A_{1}\right)$ above we see that $A$ is an irreducible curve of arithmetic genus 1 . Since $A \cdot F=1$, if we set $D:=A+F$, we will have $D^{2}=0, K \cdot D=2$. By arguing as in the proof of lemma (3.5), we see that there is a pencil $f: S \rightarrow E$ with elliptic base $E$ and such that a multiple $m D$ of $D$, with $m \geq 2$, is a curve of the pencil.

Since $|K+B|+A$ is contained in $|K+M|$ and $A \neq F$, we see, by lemma (3.3, iv), that $F$ is a fixed component of $|K+B|$. Now, since $F \leq B$ and $F \cdot(B-F)=2$, we find by $\left(0.12\right.$, a) that $B$ is not 2 -connected. Then $B=B_{1}+B_{2}$, with $B_{1} \cdot B_{2}=1$. Since $D$ is nef, by 2 -connectedness of the canonical divisors $B_{1} \cdot D \geq 1$ and $B_{2} \cdot D \geq 1$. Therefore, since $B \cdot D=2$, we must have $B_{i} \cdot D=1$ and $K \cdot B_{i}=B_{i}^{2}+2, i=1,2$.

If either $B_{1}$ or $B_{2}$ were of arithmetic genus 1 , we would have a contradiction to (0.21). Hence $K \cdot B_{i}>1, i=1,2$. Therefore, since $K \cdot B=5$, we can only have, say, $K \cdot B_{1}=2$ and $K \cdot B_{2}=3$, yielding $B_{1}^{2}=0$ and $B_{2}^{2}=1$. By $(0.9)$ there is then another pencil $g: S \rightarrow E^{\prime}$, with elliptic base $E^{\prime}$, such that $n B_{1}, n \geq 2$, is a curve of the pencil. Since $B_{1} \cdot(A+F)=B_{1} \cdot D=1$ and $B_{1}$ is nef, we get either $B_{1} \cdot A=1$ or $B_{1} \cdot F=1$, and so we again find a contradiction to $(0.21)$.

Case (b) is again excluded, since it would imply the existence of base points for $|M|$.

As for (c), it also cannot happen. In fact we may assume $F \leq A$. We find $(A-F)^{2}=-2, K \cdot(A-F)=2$ and $M \cdot(A-F)=0$. If $A-F$ is 1 -connected, then $|M-(A-F)|=|B+F|$ is a pencil. Since $K \cdot(B+F)=4$, we find a contradiction to lemma (3.2). If $A-F$ is not 1-connected, in particular it is reducible and we have $A-F=A_{1}+A_{2}$ with $M \cdot A_{i}=0, K \cdot A_{i}=F \cdot A_{i}=1, A_{i}^{2}=-1, i=1,2$; hence $A_{1} \cdot A_{2}=0$. Since there are no smooth rational curves on $S$, the curves $A_{1}$ and $A_{2}$ are irreducible curves with arithmetic genus 1 . If we put $D=F+A_{1}$, we have $K \cdot D=2, D^{2}=0$, and $A_{2} \cdot D=1$, and we find again a contradiction to $(0.21) \cdot \diamond$ 
Next we turn to the discussion of the cases $(B)$. Here the general curve in $M$ is irreducible and non-singular by lemma $(3.3$, vi). Furthermore, by $(3.3$, ii) we see that $F$ is irreducible in case $\left(B_{2}\right)$, whereas in case $\left(B_{1}\right)$ it is a fundamental cycle of type $\left(A_{i}\right), i=1,2$. In particular, if $F^{\prime}$ is an irreducible component of $F$, one has $F \cdot F^{\prime}<0$ and $M \cdot F^{\prime}>0$. Thus, by (3.1), every smooth rational curve $\theta$ in $S$ is such that $M \cdot \theta>0$.

(3.11) Lemma. If $p$ is a base point of $|K+M|$, there exists an irreducible curve $D$ passing through $p$ and satisfying $D^{2}=-1, K \cdot D=1$ and $M \cdot D=0$. Furthermore, $h^{0}\left(S, \mathcal{O}_{S}(M-2 D)\right) \neq 0$.

Proof. Suppose $p$ is a base point of $|K+M|$. Since $M^{2}=5$ and $M$ is nef, (0.13) yields the existence of a 1 -connected curve $D$ passing through $p$, such that $K \cdot(M-$ $2 D) \geq 0$ and satisfying either $M \cdot D=0$ and $D^{2}=-1$ or $M \cdot D=1$ and $D^{2}=0$. Since $K \cdot M=7$ we have the following possibilities:

$\begin{array}{ccc} & D^{2} & K \cdot D \\ \text { (a) } & -1 & 3 \\ \text { (b) } & 0 & 2 \\ \text { (c) } & -1 & 1\end{array}$

Case (a) cannot happen. In fact $D$ is 1 -connected and $M \cdot D=0$, whence $h^{0}\left(S, \mathcal{O}_{S}(M-D)\right)=2$ and $K \cdot(M-D)=4$, contradicting lemma (3.2). Also case (b) cannot happen. In fact the same argument as in lemma (3.5) yields the existence of a pencil $f: S \rightarrow B$, with $B$ a curve of genus 1 , which has $m D$, for some $m \geq 2$, as a multiple fiber. Since $D \cdot F=1$, this contradicts $(0.21)$.

Therefore only case (c) can happen. Now suppose that $D$ is not irreducible. Since $K \cdot D=1$, there will be an irreducible component of $D$ which is a (-2)-curve. But this is impossible. In fact in case $\left(B_{2}\right)$ there are no $(-2)$-curves on $S$, while in case $\left(B_{1}\right)$ every $(-2)$-curve $\theta$ in $S$ is such that $M \cdot \theta>0$. But since $M \cdot D=0$ and $M$ is nef, no $(-2)$-curve can be a component of $D$.

The curve $D$ is irreducible with $p_{a}(D)=1, D^{2}=-1$, thus $h^{0}\left(2 D, \mathcal{O}_{2 D}\right)=2$. Moreover $\mathcal{O}_{2 D} \simeq \mathcal{O}_{2 D}(M)$, and thus the last assertion is proved. $\diamond$

(3.12) Proposition. Cases $\left(B_{1}\right)$ and $\left(B_{2}\right)$ do not occur.

Proof. By lemma (3.3, iv) we know that the image of the restriction map $r$ : $H^{0}\left(S, \mathcal{O}_{S}(K+M)\right) \rightarrow H^{0}\left(F, \mathcal{O}_{F}(K+M)\right)$ is at most 1-dimensional. Since $(K+$ $M) \cdot F>0$, the linear system $|K+M|$ has at least one base point $p$ on $F$. Then by lemma (3.11) there is an irreducible curve $D$ with $D^{2}=-1, K \cdot D=1, M \cdot D=0$, $F \cdot D=1$ passing through $p$, and it is clear that $D \cap F=\{p\}$. This implies that $\operatorname{Im}(r)$ is 1-dimensional, and thus $h^{0}\left(S, \mathcal{O}_{S}(2 M)\right)=6$ : otherwise $D$ would vary, as $p$ moves on $F$, in a positive-dimensional family of irreducible curves with $D^{2}=-1$.

We first exclude that $|K+M|$ has two distinct base points $p, q$ on $F$. In fact, if this happens (3.11) implies the existence of two irreducible curves $D$ and $D^{\prime}$ passing through $p$ and $q$ respectively and such that $D^{2}=D^{\prime 2}=-1, K \cdot D=K \cdot D^{\prime}=1$ and $F \cdot D=F \cdot D^{\prime}=1$. Since $p \neq q$, also $D$ and $D^{\prime}$ are distinct. Since $D$ and $D^{\prime}$ are contracted by $\phi_{M}$, then $D \cdot D^{\prime}=0$.

Set $N=D^{\prime}+F$ in case $\left(B_{2}\right)$ and $N=D+D^{\prime}+F$ in case $\left(B_{1}\right)$. Then $N^{2}=0$ and $K \cdot N=2$, and $N$ is 1 -connected by (0.11). As usual (cf. lemma (3.5)) this yields the existence of a pencil with elliptic base of which $m N$, for some $m \geq 2$, is a fibre. 
In case $\left(B_{2}\right)$ we have a contradiction to $(0.21)$, since $D \cdot N=1$.

In case $\left(B_{1}\right)$ consider the long exact sequence obtained from

$$
0 \rightarrow \mathcal{O}_{S} \rightarrow \mathcal{O}_{S}(N) \rightarrow \mathcal{O}_{N}(N) \rightarrow 0 .
$$

From $h^{0}\left(N, \mathcal{O}_{N}(N)\right)=0$ and $q=3$ we obtain $h^{1}\left(S, \mathcal{O}_{S}(N)\right) \geq 3$. Applying the theorem of Riemann-Roch to $N$ we obtain

$$
h^{0}\left(S, \mathcal{O}_{S}(K-N)\right)+h^{0}\left(S, \mathcal{O}_{S}(N)\right)=h^{1}\left(S, \mathcal{O}_{S}(N)\right) \geq 3 .
$$

Clearly $h^{0}\left(S, \mathcal{O}_{S}(N)\right)=1$, and since $N$ is not contained in the fixed locus of $|K|$ we have $h^{0}\left(S, \mathcal{O}_{S}(K-N)\right)=2$ and $h^{1}\left(S, \mathcal{O}_{S}(N)\right)=3$. Let $B:=K-N$. We have $B^{2}=3, K \cdot B=5$, and the general curve in $|B|$ is irreducible. In fact let $\Gamma$ be the fixed part of $|B|$. By lemma (3.2) one has $K \cdot \Gamma=0$, hence $N \cdot \Gamma=0$, and therefore $B \cdot \Gamma=0$. Then $(B-\Gamma)^{2}<3$, contradicting lemma (3.2).

Since $B \cdot F=0$, there exists $B^{\prime} \in|B|$ such that $B^{\prime}=A+F$ with $A$ a curve such that $A \cdot F=2, A^{2}=1$ and $K \cdot A=5$.

Now consider the restriction map $t: H^{0}\left(S, \mathcal{O}_{S}(M+B)\right) \rightarrow H^{0}\left(M, \mathcal{O}_{M}(M+B)\right)$. Since $h^{0}\left(S, \mathcal{O}_{S}(B-M)\right)=0$, the image of the map $r_{1}: H^{0}\left(S, \mathcal{O}_{S}(B)\right) \rightarrow$ $H^{0}\left(S, \mathcal{O}_{M}(B)\right)$ has dimension equal to $h^{0}\left(S, \mathcal{O}_{S}(B)\right)=2$. Furthermore, the image of $r_{2}: H^{0}\left(S, \mathcal{O}_{S}(M)\right) \rightarrow H^{0}\left(S, \mathcal{O}_{M}(M)\right)$ has also dimension 2 . This yields that the image of $t$ is at least 3-dimensional, which implies $h^{0}\left(S, \mathcal{O}_{S}(M+B)\right) \geq 5$, whence $h^{1}\left(S, \mathcal{O}_{S}(M+B)\right) \geq 1$.

Notice that $|M+B|=|K+A|$. Hence $h^{1}\left(S, \mathcal{O}_{S}(K+A)\right)=h^{1}\left(S, \mathcal{O}_{S}(M+B)\right)>0$ and therefore $A$ is not 1 -connected (see [B], pg. 178). Then, since $A \cdot F=2$ and $B$ is 1-connected (see (0.11)), one obtains $A=A_{1}+A_{2}$, where $A_{1}, A_{2}$ are curves such that $A_{1} \cdot A_{2}=0, A_{1} \cdot F=A_{2} \cdot F=1$. By 2-connectedness of the canonical divisors we have $2 \leq A_{i} \cdot\left(K-A_{i}\right)=A_{i} \cdot F+A_{i} \cdot N$. Since $B$ is nef we get $A_{i} \cdot B \geq 0$, whence $A_{i}^{2} \geq-1$, for $i=1,2$, and therefore we have either, say, $A_{1}^{2}=-1, A_{2}^{2}=2$ or $A_{1}^{2}=0, A_{2}^{2}=1$. We saw that $K \cdot A_{i} \geq A_{i}^{2}+2, i=1,2$, and $K \cdot A=5$. Therefore in the first case $K \cdot A_{1}=1$ and in the second case $K \cdot A_{1}=2$. This contradicts (0.21) in both cases. In the first case we would have $M \cdot A_{1}=0$, and therefore $A_{1}$ is irreducible, since $M \cdot \theta>0$ for every (-2)-curve $\theta$ on $S$, and in addition $A_{1} \cdot N=1$. In the second we would obtain a second irrational pencil with elliptic base, with $m A_{1}$, for some $m \geq 2$, a curve of the pencil, and in addition $A_{1} \cdot F=1$.

We have proved so far that $|K+M|$ cannot have two distinct base points on $F$. Whence, we may now turn to the case in which there is only one base point for $|K+M|$. Let $p$ be this base point lying on $F$ and let $D$ be the curve as in (3.11) passing through $p$. Now every divisor in $|M|$ is 1-connected and $(M-D) \cdot D=1$, every divisor in $|M-D|$ is 1 -connected (see $(0.11)$ ), and $(M-D)^{2}>0$. Thus $h^{1}\left(S, \mathcal{O}_{S}(K+M-D)\right)=0$ and by Riemann-Roch $h^{0}\left(S, \mathcal{O}_{S}(K+M-D)\right)=6$. Since $h^{0}\left(S, \mathcal{O}_{S}(2 M-D)\right)=5$, the image of the restriction map

$$
\rho: H^{0}\left(S, \mathcal{O}_{S}(K+M-D)\right) \rightarrow H^{0}\left(F, \mathcal{O}_{F}(K+M-D)\right)
$$

is 1-dimensional and therefore $|K+M-D|$ has base points on $F$. Let $s$ be the restriction to $F$ of the section in $H^{0}\left(S, \mathcal{O}_{S}(D)\right)$, which is non-zero on $F$ and vanishes only at $p$. Then $s \cdot \operatorname{Im}(\rho)$ is 1-dimensional and is contained in $\operatorname{Im}(r)$, which, as we saw, is also 1 -dimensional. Hence $s \cdot \operatorname{Im}(\rho)=\operatorname{Im}(r)$. Since the only point of $F$ where the sections in $\operatorname{Im}(r)$ all vanish is $p$, then the same happens for the sections in $\operatorname{Im}(\rho)$, i.e. $p$ is the unique base point of $|K+M-D|$ on $F$. Since $(K+M-D) \cdot D=2$, this implies that also the image of the restriction map 
$r^{\prime}: H^{0}\left(S, \mathcal{O}_{S}(K+M-D)\right) \rightarrow H^{0}\left(D, \mathcal{O}_{D}(K+M-D)\right)$ is at most 1-dimensional, and therefore $h^{0}\left(S, \mathcal{O}_{S}(K+M-2 D)\right) \geq 5$. Now let $A:=M-2 D$. Since $A^{2}=1$ and $K \cdot A=5$, Riemann-Roch implies $h^{1}\left(S, \mathcal{O}_{S}(K+M-2 D)\right)>0$; thus $A$ is not 1-connected. So $A=A_{1}+A_{2}$, where $A_{1} \cdot A_{2} \leq 0$. Since every divisor in $|M|$ is 1connected, we have $A_{i} \cdot D \geq 1$, and therefore $A_{i} \cdot D=1$ because $D \cdot A=2$. Then one has $A_{1} \cdot A_{2}=0$ : otherwise we would have $\left(A_{1}+D\right) \cdot\left(A_{2}+D\right) \leq 0$, contradicting the connectedness of $M$. By 2-connectedness of the canonical divisors we find $A_{i} \cdot F \geq 0, i=1,2$, and from $A \cdot F=0$ we deduce $A_{i} \cdot F=0, i=1,2$. Furthermore $A_{i}^{2}=\bar{A} \cdot A_{i}=K \cdot A_{i}-2$, and $K \cdot A_{i} \geq 1$ since $A_{i} \cdot F=0, i=1,2$. Hence $A_{i}^{2} \geq-1$, $i=1,2$, and if, say, $A_{1}^{2}=0, A_{2}^{2}=1$, we contradict the index theorem. So we can assume that $A_{1}^{2}=-1, K \cdot A_{1}=1$.

This gives rise to a contradiction to (0.21). In fact $m\left(A_{1}+D\right)$, for some $m \geq$ 2 , would be a multiple fibre of a pencil with elliptic base (see lemma (3.5)) and $\left(A_{1}+D\right) \cdot F=1 . \diamond$

Our discussion of cases $(A)$ and $(B)$ is thus concluded.

Case $\phi$ not birational, $K^{2}=8$, and $|K|$ with no fixed component.

Let us assume $\phi$ not birational, $|K|$ with no fixed component, $K^{2}>6$. By proposition $(3.1, \mathrm{v})$ we have then $K^{2}=8$, and by $(0.1)$ the general canonical curve is irreducible and reduced. Furthermore, by lemma (3.1, iii), on $S$ there are no rational curves at all.

(3.13) Lemma. Let $p_{g}=q=3, K^{2}=8$, and let $|K|$ have no fixed components. If $\phi$ is not birational, then $\sigma=2$.

Proof. Assume, to the contrary, that $\sigma=4$. Let $C$ be the general curve in $|K|$. Then $\phi(C)$ would have degree 4 and it should span a $\mathbf{P}^{5}$, a contradiction. $\diamond$

From lemma (3.13) we deduce the existence of the bicanonical involution $\iota$ (cf. the case $q=1$ in the proof of proposition (2.1)). Notice that $\iota$ acts as the identity on $|K|$ and $|2 K|$.

(3.14) Lemma. Let the bicanonical map $\phi$ have degree 2 , and assume that $q \geq 3$ and there is no irrational pencil on $S$ of genus at least 2. Then, if $i$ is the involution associated to $\phi$, then $i^{*}$ acts as +1 on $H^{0}\left(\mathcal{O}_{S}(K)\right)$.

Proof. $i^{*}$ on $H^{0}\left(\Omega_{S}^{1}\right)$ has an eigenspace of dimension $\geq 2$. Thus we get two linearly independent 1 -forms whose exterior product is non zero, and yields a $(+1)$ eigenvector in $H^{0}\left(\mathcal{O}_{S}(K)\right)$. We conclude since by our assumption we knew that $i^{*}$ would act either as +1 or as -1 on $H^{0}\left(\mathcal{O}_{S}(K)\right)$. $\diamond$

(3.15) Lemma. Same assumptions as in lemma 1 , and let $Y=S / i, p: S \rightarrow Y$ the double cover. Then $p$ is unramified in codimension 1 if the canonical system of $S$ has no fixed part.

Proof. Let $R$ be the ramification divisor of $p$. We have an inclusion $R+p^{*}\left|K_{Y}\right|$ contained in $\left|K_{S}\right|$. But both systems have the same dimension, whence the assertion easily follows. $\diamond$

(3.16) Proposition. Let $p_{g}=q=3, K^{2}=8$, and let $|K|$ have no fixed component. Then, if $\phi$ is not birational, $S$ presents the standard case.

Proof. Since $\left|K_{S}\right|$ has two simple base points, $\left|K_{Y}\right|$ has exactly one base point, which is not a ramification point. Then the involution $i$ acts freely on the general 
canonical curve $C$ of $S$. Thus $C=C / i$ has genus 5 . But its image $\phi(C)$, birational to $C$, has degree 8 and should span a $\mathbf{P}^{5}$ : this is a contradiction. $\diamond$

The case $K^{2}=6$.

Now we examine the case $K^{2}=6$. First we prove

(3.17) Proposition. Let $C$ be a smooth projective connected curve of genus 3. Then its symmetric product $S=C^{(2)}$ is a smooth minimal surface with $p_{g}=q=3$, $K^{2}=6$, such that the bicanonical map is not birational, of degree 2. Furthermore:

(i) if $C$ is not hyperelliptic, then the canonical system $|K|$ of $S$ is base point free and the bicanonical involution sends a divisor $x+y$ to $\left|K_{C}-x-y\right|$;

(ii) if $C$ is hyperelliptic, then the canonical system $|K|=F+|M|$ is of the type (D) of the table in lemma (3.3, iii). Then the fixed part $F$ of $|K|$ is the rational (-2)-curve described by the divisors of the $g_{2}^{1}$, and the movable part $|M|$ is base point free. The bicanonical involution sends a divisor $x+y$ to the divisor $j(x)+j(y)$, where $j: C \rightarrow C$ is the bicanonical involution;

(iii) in either case $S$ presents the non-standard case.

Proof. It is well known that $q=3$, and $\operatorname{Alb}(S) \simeq J(C):=P i c^{0}(C)$. If one fixes a theta-characteristic $\kappa$ on $C$, the Albanese morphism coincides with the Abel-Jacobi map $a: x+y \in C^{(2)} \rightarrow \mathcal{O}_{C}(x+y-\kappa) \in J(C)$. The image of $C^{(2)}$ via $a$ is a symmetric theta divisor $\Theta$ which determines a principal polarization of $J(C)$. Notice that $\Theta$ is smooth if and only if $C$ is not hyperelliptic, whereas, if $C$ is hyperelliptic, it has a double point which comes from the contraction of the rational $(-2)$-curve $F$ described by the divisors of the $g_{2}^{1}$, which is the only rational curve on $C^{(2)}$. In any event $\Theta$ is nothing but the canonical model of $S$.

From the exact sequence

$$
0 \rightarrow \mathcal{O}_{J(C)} \rightarrow \mathcal{O}_{J(C)}(\Theta) \rightarrow N_{\Theta, J(C)} \simeq \omega_{\Theta} \rightarrow 0
$$

and from the fact that $h^{0}\left(J(C), \mathcal{O}_{J(C)}(\Theta)\right)=1$ and $h^{1}\left(J(C), \mathcal{O}_{J(C)}(\Theta)\right)=0$, we see that $p_{g}=h^{0}\left(\Theta, \omega_{\Theta}\right)=h^{1}\left(J(C), \mathcal{O}_{J(C)}\right)=3$. Furthermore the exact sequence

$$
0 \rightarrow \mathcal{O}_{J(C)}(\Theta) \rightarrow \mathcal{O}_{J(C)}(2 \Theta) \rightarrow N_{\Theta, J(C)}^{\otimes 2} \simeq \omega_{\Theta}^{\otimes 2} \rightarrow 0
$$

shows that the map

$$
H^{0}\left(J(C), \mathcal{O}_{J(C)}(2 \Theta)\right) \rightarrow H^{0}\left(\Theta, \omega_{\Theta}^{\otimes 2}\right)
$$

is surjective. Since the linear system $|2 \Theta|$ is composite with the involution induced by the natural action of $\mathbf{Z}_{2}$ on $J(C)$, the same happens for $\left|\omega_{\Theta}^{\otimes 2}\right|$ on $\Theta$, which is symmetric, i.e. stable for the $\mathbf{Z}_{2}$-action. This proves the first assertion.

By proposition $(3.1, \mathrm{i})$ there is no irrational pencil on $S$, which proves (iii).

Let us look now at the following chain of isomorphisms:

$$
\begin{gathered}
H^{0}\left(C, \mathcal{O}_{C}\left(K_{C}\right)\right)^{*} \simeq \wedge^{2} H^{0}\left(C, \mathcal{O}_{C}\left(K_{C}\right)\right) \simeq \wedge^{2} H^{0}\left(J(C), \Omega_{J(C)}^{1}\right) \\
\simeq H^{0}\left(J(C), \Omega_{J(C)}^{2}\right) \stackrel{a^{*}}{\longrightarrow} H^{0}\left(C^{(2)}, \Omega_{C^{(2)}}^{2}\right)
\end{gathered}
$$

which gives rise to a projective transformation

$$
f: \mathbf{P}\left(H^{0}\left(C, \mathcal{O}_{C}\left(K_{C}\right)\right)^{*}\right) \rightarrow\left|K_{C^{(2)}}\right| .
$$


In geometric terms, $f$ is described as follows. A point of $\mathbf{P}\left(H^{0}\left(C, \mathcal{O}_{C}\left(K_{C}\right)\right)^{*}\right)$ is a linear series $\xi$ of dimension 1 contained in $\left|K_{C}\right|$. Let $T_{\xi}$ be the curve on $C^{(2)}$ described by all divisors $x+y$ such that $x+y<D \in \xi$. The map $f$ sends $\xi$ to $T_{\xi}$. With this description of the canonical curves on $S$ in mind, assertions (i) and (ii) are clear. Notice that the fact that $|M|$ is base point free in part (ii) also follows from $(3.1, \mathrm{i})$ and $(3.3, \mathrm{v}) . \diamond$

(3.21) Remark. The properties of the canonical system of $C^{(2)}$ could also be deduced from the isomorphism $H^{0}\left(\Theta, N_{\Theta, J(C)}\right) \simeq H^{0}\left(\Theta, \omega_{\Theta}\right)$. In fact in the hyperelliptic case $\Theta$ is singular with all its translates. Hence all its infinitesimal deformations, i.e. all sections of $H^{0}\left(\Theta, N_{\Theta, J(C)}\right)$, vanish at the singular point, showing that $|K|$ has the fixed component as stated in proposition (3.20). On the other hand, in the non-hyperelliptic case $\Theta$ is smooth, and it is clear that the infinitesimal deformations do not vanish simultaneously at any point of $\Theta$, showing that $|K|$ is base point free on $C^{(2)}$.

Now we are ready to prove the following proposition:

(3.22) Proposition. Let $S$ be a surface with $p_{g}=q=3$ and $K^{2}=6$. Then $S$ is the symmetric product of a curve of genus 3.

Proof. First we assume that $|K|$ has no fixed components. By proposition (3.1, iii, iv) the Albanese map $a: S \rightarrow S^{\prime} \subseteq A l b(S)$ is unramified. Then proposition (4.1) of [De2] implies that $a$ is an embedding.

Let us write $S^{\prime} \sim i D$, where $D$ is an effective indivisible divisor on $A l b(S)$, determining a polarization of type $\left(1, d_{1}, d_{2}\right)$. Then $6=K^{2}=S^{\prime 3}=i^{3} D^{3}=$ $6 d_{1} d_{2} i^{3}$, which implies $i=d_{1}=d_{2}=1$, proving our assertion. In fact a smooth theta divisor on a principally polarized abelian threefold is the symmetric product of a curve of genus three.

Assume now that $|K|$ has a fixed component. By lemma $(3.3, \mathrm{i})$ we are in case $(C)$ of the table in the same lemma (3.3, iii). We have $|K|=F+|M|$, with $F$ a fundamental cycle. By lemma $(3.3, \mathrm{v})$, the linear system $|M|$ is base point free. Let $\pi: S \rightarrow X$ be the contraction of $F$ which gives rise to a normal surface $X$, and let $x$ be the singular point of $x$. The Albanese map $a: S \rightarrow S^{\prime} \subseteq \operatorname{Alb}(S)$ factors through $\pi$ and through a map $b: X \rightarrow S^{\prime} \subseteq A l b(S)$. The map $b$ is unramified at the smooth points of $X$ because $|M|$ is base point free. In order to again apply Debarre's result to $b$, thus proving the assertion, we have to prove that $b$ is also unramified at $x$.

Suppose $b$ is ramified at $x$. Note that the codifferential of $b$ at $x$ is the map

$$
b^{*}: H^{0}\left(A l b(S), \Omega_{A l b(S)}^{1}\right) \simeq H^{0}\left(S, \Omega_{S}^{1}\right) \rightarrow \Omega_{X}^{1} / \mathbf{m}_{x} \Omega_{X}^{1},
$$

where $\Omega_{X}^{1}$ is the sheaf of Kaehler differentials of $X$ and $\mathbf{m}_{x}$ is the maximal ideal of $X$ at $x$. Note that $H^{0}\left(S, \Omega_{S}^{1}\right) \simeq H^{0}\left(X, \Omega_{X}^{1}\right)$ via the map $\pi^{*}$. Then $b$ ramified at $x$ means that there is a non-zero form $\eta \in H^{0}\left(X, \Omega_{X}^{1}\right)$ which is zero at $x$. Let $\eta_{1}$ and $\eta_{2}$ be forms in $H^{0}\left(X, \Omega_{X}^{1}\right)$ forming with $\eta$ a basis of $H^{0}\left(X, \Omega_{X}^{1}\right)$. Then the 2 -forms $\omega_{i}=\eta \wedge \eta_{i}, i=1,2$, are linearly independent elements of $H^{0}\left(X, \Omega_{X}^{2}\right)$, which both vanish at $x$.

Now we observe that if $\omega \in H^{0}\left(X, \Omega_{X}^{2}\right)$ vanishes at $x$, then the form $\pi^{*}(\omega)$ vanishes with multiplicity two along $F$. This is an obvious consequence of the following remarks: 
(i) A rational double point is locally embedded in $\mathbf{C}^{3}$ with coordinates $\left(x_{1}, x_{2}, x_{3}\right)$. Then a 2 -form vanishing at $x$ is of the type $\omega=\sum_{1 \leq i<j \leq 3} a_{i j} d x_{i} \wedge d x_{j}$, with $a_{i j}$ holomorphic functions vanishing at $x$.

(ii) A rational double point can be resolved with a finite number of blowing-ups with centres at rational double points.

(iii) Any holomorphic 2-form on $\mathbf{C}^{3}$ pulls back, on the blow-up of $\mathbf{C}^{3}$ at a point, to a form vanishing along the exceptional divisor.

In conclusion we have that $b$ ramified at $x$ would yield $h^{0}\left(S, \mathcal{O}_{S}(K-2 F)\right)=$ $h^{0}\left(S, \mathcal{O}_{S}(M-F)\right)=2$. This in turn would imply that $|M|$ has two base points on $F$, against lemma $(3.3, \mathrm{v}) . \diamond$

The standard case.

Finally we classify the standard cases with $p_{g}=q=3$. Actually, improving on proposition $(3.1, \mathrm{i})$, we prove the following more general result:

(3.23) Theorem. Let $S$ be a minimal surface of general type with $p_{g}=q=3$ and an irrational pencil $f: S \rightarrow B$, with $B$ a smooth curve of genus $b \geq 1$ and fibres of genus $g$. Then either $b=1$ and $g \geq 3$ or $b=g=2, K^{2}=8$, the map $f$ is smooth and the curves $F$ of the pencil have constant modulus. The latter case happens if and only if there is a commutative diagram

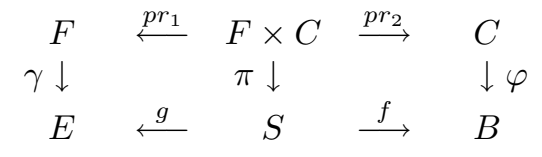

where $\varphi: C \rightarrow B$ and $\gamma: F \rightarrow E$ are double covers, the first unramified with $C$ smooth of genus 3, the latter ramified with $E$ smooth of genus 1 , and $\pi: F \times C \rightarrow S$ is the unramified double cover obained by pulling back $f$ via $\varphi$. In other words, there exist a smooth irreducible curve $C$ of genus 3 and an action of $G=\mathbf{Z} / 2 \mathbf{Z}$ on $C$ and $F$, free on $C$, such that $B \simeq C / G$ and $S \simeq F \times C / G$, where $G$ acts diagonally on $F \times C$.

Proof. The first assertion is $(3.1, \mathrm{i})$. Let us assume now that $b=g=2$. Then $\operatorname{Aut}(F)$ is finite and $f$ is given by a representation $\rho: \pi(B) \rightarrow \operatorname{Aut}(F)$. Hence $\operatorname{ker}(\rho)$ corresponds to a finite, unramified, Galois cover $\varphi: C \rightarrow B$ with Galois group $G:=\operatorname{Im}(\rho)$, such that the pull-back of $f$ via $\varphi$ is a product

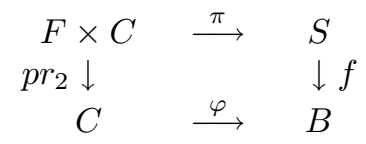

The group $G$ acts on both $C$ and $F$, and clearly

$$
\begin{gathered}
H^{0}\left(S, \Omega_{S}^{1}\right) \simeq H^{0}\left(F \times C, \Omega_{F \times C}^{1}\right)^{G} \simeq H^{0}\left(C, \Omega_{C}^{1}\right)^{G} \oplus H^{0}\left(F, \Omega_{F}^{1}\right)^{G} \\
\simeq H^{0}\left(B, \Omega_{B}^{1}\right) \oplus H^{0}\left(E, \Omega_{E}^{1}\right)
\end{gathered}
$$

where $E=F / G$. Therefore, as $q=3, b=2$, the curve $E$ has genus 1 . Let us consider the cover $\gamma: F \rightarrow E$ of order $m$ equal to the order of $G$. Suppose that $\gamma$ has $k$ branch points $x_{1}, \ldots, x_{k}$, and that $\gamma$ has a ramification point of order $m_{i}$ at any one of the $m / m_{i}$ points above $x_{i}$, for any $i=1, \ldots, k$. The Hurwitz formula tells us that 


$$
2=\sum_{i=1}^{k} \frac{m}{m_{i}}\left(m_{i}-1\right) .
$$

This immediately implies that $m_{i} \leq 3$ for all $i=1, \ldots, k$, and one has the following possibilities: either $k=1, m=m_{1}$, or $k=1, m=4, m_{1}=2$, or $k=2, m=$ $m_{1}=m_{2}=2$. In any case $G$ is abelian, and we claim that this implies that $\gamma$ cannot be branched at only one point $x$. Otherwise $\gamma$ would yield a representation $\pi_{1}(E-\{x\}) \simeq \mathbf{Z} \star \mathbf{Z} \rightarrow G$ which would factor through $\pi_{1}(E) \simeq \mathbf{Z} \times \mathbf{Z} \rightarrow G$, implying that $\gamma$ is unramified, a contradiction. Thus the only possibility is $k=2$, $m=m_{1}=m_{2}=2$, proving the assertion.

Finally, notice that if we have two smooth irreducible curves $C$ and $F$, the former of genus 3, the latter of genus 2 , and an action of $G=\mathbf{Z} / 2 \mathbf{Z}$ on $C$ and $F$, free on $C$, such that $B:=C / G$ has genus 2 and $E:=F / G$ has genus 1 , then $G$ acts diagonally on $F \times C$ and the action is free. The quotient $S \simeq F \times C / G$ has $q=3$ by (3.24) and $\chi\left(\mathcal{O}_{S}\right)=\frac{1}{2} \chi\left(\mathcal{O}_{F \times C}\right)=1$, whence $p_{g}=q=3 . \diamond$

\section{REFERENCES}

[B] E. Bombieri, Canonical models of surfaces of general type, Publ. Math. IHES, 42 (1973), 171-219. MR 47:6710

[Be1] A. Beauville, L'inégalité $p_{g} \geq 2 q-4$ pour les surfaces de type générale, Appendix to [De1], Bull. Soc. Math. France, 110 (1982), 343-346. MR 84f:14026

[Be2] A. Beauville, Annulation du $H^{1}$ et systémes paracanoniques sur les surfaces J. Reine Angew. Math. 388 (1988), 149-157. MR 81i:14032

[BPV] W. Barth, C. Peters, A. Van de Ven, Compact Complex Surfaces, Ergebnisse der Math. (3) 4, Springer-Verlag, 1984. MR 86c:32026

[BS] M. Beltrametti, A. Sommese, Zero-cycles and kth order embeddings of smooth projective surfaces, in Problems in the Theory of Surfaces and Their Classification (F. Catanese, C. Ciliberto, M. Cornalba, ed.), Symposia Math. (INDAM) 32 (1991), 33-48. MR 95d:14005

[Ca] F. Catanese, On the moduli spaces of surfaces of general type, J. Differential Geometry, 19 (1984), 483-515. MR 86h:14031

[CFM] C. Ciliberto, P. Francia, M. Mendes Lopes, Remarks on the bicanonical map for surfaces of general type, Math.Z. 224 (1997), 137-166.

[D] P. Du Val, On surfaces whose canonical system is hyperelliptic, Canadian J. Math., 4 (1952), 204-221. MR 13:977e

[De1] O. Debarre, Inégalités numériques pour les surfaces de type general, Bull. Soc. Math. de France, 110 (1982), 319-346. MR 84f:14026

[De2] O. Debarre, Théorèmes de connexité et variétés abéliennes, Amer. J. Math 117 (1995), 787-805. MR 96j:14009

[GL] M. Green, R. Lazarsfeld, Higher obstructions to deforming cohomology groups of line bundles, J. Amer. Math. Soc. 4 (1991), 87-103. MR 92i:32021

[Ho] E. Horikawa, Algebraic surfaces of general type with small $c_{1}^{2}$, J. Fac. Sci. Univ. Tokyo, Sec. IA, Math. 28 (1981), 745-755. MR 84d:14019

[M] M. Mendes Lopes, Adjoint systems on surfaces, Boll. Unione Mat. Italiana A(7) 10 (1996), 169-179. CMP 96:11

[Mi] Y. Miyaoka, The maximal number of quotient singularities on surfaces with given numerical invariants, Math. Ann. 268 (1984), 159-171. MR 85j:14060

[P] G. Pirola, Curves on Kummer varieties, pre-print.

[Ra] C. P. Ramanujam, Remarks on the Kodaira vanishing theorem, J. Indian Math. Soc. 36 (1972), 41-51, suppl. ibidem 38 (1974), 121-124. MR 48:8502; MR 52:13859

[R] I. Reider, Vector bundles of rank 2 and linear systems on algebraic surfaces, Ann. of Math. 127 (1988), 309-316. MR 89e:14038

[X1] G. Xiao, Degree of the bicanonical map of a surface of general type, Amer. J. Math., 112 (5) (1990), 713-737. MR 91i:14030 
[X2] G. Xiao, L'irrégularité des surfaces de type général dont le systéme canonique est composé d'un pinceau, Compositio Math. 56 (1985), 251-257. MR 87d:14031

[X3] G. Xiao, Irregularity of surfaces with a linear pencil, Duke Math. J., 55 (1987), 597-602. MR 89c: 14055

Dipartimento di Matematica, Università di Pisa, Via Buonarroti 2, 56127 Pisa, Italy Current address: Mathematisches Institut der Georg-August, Universität Göttingen, Bunsenstraße 3-5, D-37073 Göttingen, Germany

E-mail address: catanese@uni-math.gwdg.de

Dipartimento di Matematica, Università di Tor Vergata, Viale della Ric. ScienTIFICA, 16132 Roma, ITALY

E-mail address: cilibert@axp.mat.utovrm.it

Dipartimento di Matemática, Faculdade de Ciencias de lisboa, R. Ernesto de VasCONCElos, 1700 Lisboa, Portugal

E-mail address: mmlopes@ptmat.lmc.fc.ul.pt 